\title{
A Present Day Perspective on Einstein-Podolsky-Rosen and Its Consequences
}

\author{
Jeremy Dunning-Davies ${ }^{1,2}$ \\ ${ }^{1}$ Departments of Mathematics and Physics (retd), University of Hull, Hull, England \\ ${ }^{2}$ Institute for Basic Research, Palm Harbor, Florida, USA \\ Email:masjd@masjd.karoo.co.uk
}

How to cite this paper: Dunning-Davies, J. (2021) A Present Day Perspective on Einstein-Podolsky-Rosen and Its Consequences. Journal of Modern Physics, 12, 887-936. https://doi.org/10.4236/jmp.2021.127056

Received: April 3, 2021

Accepted: May 18, 2021

Published: May 21, 2021

Copyright $\odot 2021$ by author(s) and Scientific Research Publishing Inc. This work is licensed under the Creative Commons Attribution International License (CC BY 4.0).

http://creativecommons.org/licenses/by/4.0/ (c) (i) Open Access

\begin{abstract}
Ever since it first appeared in 1935, the famous paper by Einstein, Podolsky and Rosen, questioning the completeness of quantum mechanics as a theory, has courted controversy. The initial arguments with Bohr have never been forgotten or gone away; the ideas of Bell have remained; many experiments have been performed purporting to support the stance of Bohr. More recently, however, an experiment performed by a group in Basel has questioned this accepted position and, theoretically, this new perspective has received support from at least two sources. It is the work behind these two sources, especially the second, together with the experimental work at Basel, which form the basis for this examination of the present position as far as this extremely important position for physical science is concerned. Needless to say, considering the views expressed in these two approaches, it is also necessary and appropriate to consider some possible consequences if this new view becomes accepted. Due to the fact that the recent support for the Einstein, Podolsky, Rosen argument makes use of results in iso-mathematics, iso-mechanics and iso-chemistry, these possible consequences include the exact representation of nuclear data, the achievement of an attractive force between identical valence electrons with the ensuing exact representation of molecular data, the prediction of new clean energies and the prediction of the possible recycling of nuclear waste via stimulated decay-none of which is allowable utilising traditional quantum mechanics. Hence here, as well as discussing the resolution of the long standing issue provoked by the well-known Einstein, Podolsky, Rosen article, some of these consequences will be discussed with a view to provoking more general, open-minded discussion within the scientific community.
\end{abstract}

\section{Keywords}

Einstein-Podolsky-Rosen Issue, Mayants’ Approach, Basel Experiment, 
Hadronic Mechanics

\section{Introduction}

The question of uncertainty affects many areas, including my own particular field of interest-thermodynamics, although, in that case, the effect may be felt indirect. For a moment consider the situation in thermodynamics. In traditional classical thermodynamics there are no uncertainties; all the variables, for example the internal energy and total number of particles, possess definite values. However, when systems composed of a large number of particles are to be considered, the methods of statistical mechanics have to be employed due to our present state of knowledge. As a consequence, when incorporated into thermodynamics, the realm known as statistical thermodynamics is entered. This is, in some crucial ways, totally different from classical thermodynamics because the introduction of statistical techniques has introduced uncertainty into the picture. No longer are there definite values for the internal energy or total number of particles; rather average values are considered. These average values, as with the average values of other thermodynamic variables, can fluctuate in this new regime. Hence, a degree of uncertainty is introduced which leads to the derivation of thermodynamic uncertainty relations. It is important to note, though, that these relations have been introduced via the recourse to statistical methods to describe details of the system under consideration. They have been introduced because, in a system composed of a large number of particles, it is not possible to write down all the equations of motion of the individual particles, let alone solve the resulting set of simultaneous equations. The uncertainty, therefore, has been introduced as a result of our inability to solve the exact problem; there is no inherent uncertainty in the original system, just as there is no inherent uncertainty in traditional classical thermodynamics. This reasoning follows for all statistical thermodynamic theories and indicates a very real difference between classical and statistical thermodynamics.

Indeed, the same reasoning may be seen to apply to many, if not all, problems considered utilising probability theory. For example, in introducing probability, it is popular to consider the tossing of a coin. If the coin is simply tossed, the outcome when it lands-head or tails-is totally uncertain. However, this is not so if someone is in possession of all the initial conditions pertaining to the toss. If the initial speed is known, the height to which the coin rises may be found, as may the time taken to reach that height. Similarly, the time taken to fall back to a given level may be found. If the rate of rotation is also known, that, together with the total time of flight, should enable the state of the coin on reaching the desired final level to be ascertained. Hence, the uncertainty associated with this problem really arises through a lack of knowledge of the initial conditions in the problem; it is not an inherent property of the actual system. 
It may be seen, therefore, that neither statistical thermodynamics nor probability may be termed complete theories in the sense that neither provides exact solutions to problems. In both, uncertainty is introduced as a result of the inability to write down and solve a set of exact equations and/or a lack of knowledge of initial conditions.

The above may be viewed as simple, even naïve, reflections but it must be remembered always that quantum theory is entirely probabilistic and it might be wondered if thoughts similar to those above about the nature of probability and statistical methods influenced Einstein's thinking since, in so many cases, people have recourse to probabilistic and/or statistical methods to compensate either for a lack of knowledge in a problem or for a lack of ability to solve the exact problem with which they are faced. Whatever his reason, it seems Einstein remained distrustful of the completeness of quantum mechanics as a physical theory and, in 1935, his reservations became fully apparent in his article written with the collaboration of Boris Podolsky and Nathan Rosen.

\section{Einstein, Podolsky and Rosen.}

It seems that, from the moment it first appeared in 1935, the now famous paper by Einstein, Podolsky and Rosen [1], entitled "Can Quantum-Mechanical Description of Physical Reality be Considered Complete?", has courted controversy and that still appears to be the case today. This is not surprising since, as its title clearly states, it predicted that "quantum mechanics is not a complete theory" and this because, in what followed, it was claimed that determinism could be recovered, at least under certain conditions. If contemporary accounts [2] are to be believed, the immediate response from several eminent scientists was almost one of fury. Apparently Pauli was one who was particularly annoyed, partly because the article was published in an American journal and he was concerned in case it turned the American public against the quantum theory. However, it is claimed that, in Copenhagen, Bohr was in a state of shock as well as anger and reacted by abandoning all projects on which he was working to address the problems initiated by the Einstein, Podolsky, Rosen paper. Bohr eventually devoted three months of intense work to construct a rebuttal which he published in the same journal that Einstein, Podolsky and Rosen had used - Physical Review. At the time it seemed this might be the end of the affair with scientists either convinced by Bohr's reply or feeling the whole issue to be philosophical rather than physical; it seemed experimental results were not in question and so the whole issue could be quietly forgotten.

However, precisely what did the Einstein, Podolsky, Rosen article say about physics and physical systems? It claimed that a property of a physical system is an element of physical reality if it can be predicted accurately without disturbing the system. The example cited consisted of two particles that are linked together and it was used to show that the position and momentum of a given particle may be found by taking suitable measurements of the second particle without the first 
being disturbed. Thus, it was claimed that both attributes of the first particle are elements of physical reality. However, quantum mechanics does not allow this and so the contention was that it could not be a complete theory. In fact, it was declared in the article that

"if without in any way disturbing a system we can predict with certainty (i.e. with probability equal to unity) the value of a physical quantity, then there exists an element of physical reality corresponding to this physical quantity. It seems to us that this criterion, while far from exhausting all possible ways of recognising a physical reality, at least provides us with one such way, whenever the conditions set down in it occur."

This quote has been used on numerous occasions since it first appeared but is, nevertheless, well worth repeating here in order to provide a more complete, if brief, background to the whole set of questions surrounding this important paper by one of the last century's most influential scientists. It is also worth reiterating what followed in the original article.

Actually the authors considered two particles, 1 and 2, with respective momentum and position coordinates $\left(p_{1}, q_{1}\right)$ and $\left(p_{2}, q_{2}\right)$ in a state with definite total momentum $P=p_{1}+p_{2}$ and definite relative distance $Q=q_{1}+q_{2}$. Since $P$ and $Q$ commute, this is possible. The particles are allowed to interact before observations are made on particle 1 long after such interaction has ceased. If $p_{1}$ is measured, it follows that $p_{2}$ is known without particle 2 having been disturbed. An immediate consequence of this is that, in the language of the original article, $p_{2}$ is an element of reality. If $q_{1}$ is then measured, $q_{2}$ is known without particle 2 being disturbed in any way and so $q_{2}$ is also an element of reality. Hence, the conclusion of the thought experiment is that both $p_{2}$ and $q_{2}$ are elements of reality. However, according to quantum mechanics both cannot be elements of reality simultaneously. The conclusion which followed, therefore, was that quantum mechanics was not complete.

Following the above quote, the authors spent time describing entangled states and their argument fundamentally reduced to a description of quantum entanglement for position and momentum before concluding by saying:

"Thus, by measuring either A or B, we are in a position to predict with certainty, and without in any way disturbing the second system, either the value of the quantity $\mathrm{P}$ or the value of the quantity $\mathrm{Q}$. In accordance with our criterion for reality, in the first case we must consider the quantity $\mathrm{P}$ as being an element of reality, in the second case the quantity $\mathrm{Q}$ is an element of reality. But as we have seen, both wave functions belong to the same reality. Previously we proved that either (1) the quantum-mechanical description of reality given by the wave-function is not complete, or (2) when the operators corresponding to the two physical quantities do not commute the two quantities cannot have simultaneous reality.... We are thus forced to conclude that the quantum-mechanical description of physical reality given 
by wave functions is not complete."

Here, in the quote, a slightly different notation was used from that above but this does not detract from the argument presented but the earlier notation enables in some ways a clearer linking up with what follows shortly when the later work of David Bohm is considered.

In all this, Einstein, Podolsky and Rosen made an assumption of locality, something which appears entirely reasonable; what happens in one place doesn't immediately affect what happens in another. However, the conclusion of the Einstein, Podolsky, Rosen argument definitely appears to contradict conventional quantum mechanics which asserts that the pair of particles involved in the thought experiment is described by a wave-function that only yields probabilistic predictions and simply cannot yield exact values of either the position or momentum of either particle. It also appears to contradict another quantum mechanical belief that the position and momentum of a particle may not have definite values ascribed to them simultaneously as that would violate the Heisenberg uncertainty principle. In his rebuttal, Bohr claimed he felt the trend of the Einstein, Podolsky, Rosen argument didn't appear to meet the actual situation faced in atomic physics adequately. He argued that the paradox didn't present a practical challenge to the application of quantum mechanics to real physical problems and, at the time, most physicists seemed to accept this. It might be noted, though, that this 1935 paper contains neither a paradox nor any logical flaw; rather it concludes that objective reality is incompatible with quantum mechanics being complete. It is perhaps worth remembering that Bohr harboured a deep concern over the use of language in the interpretation of quantum mechanics. For example, he advocated the use of the word "phenomenon" to refer exclusively to observations obtained under specified circumstances, including an account of the whole experiment. [3] Einstein, however, believed that a deeper theoretical framework which allowed the description of phenomena independently of these conditions should be sought and this is what he meant by the term objective reality.

Of course, it must be remembered always that the Einstein, Podolsky, Rosen argument is based on a thought experiment and thought experiments are just that thought experiments. As such they are very difficult to interpret due to the assumptions made not always being totally clear, possibly not even to the originators themselves. In fact, in a purely thought experiment, it is easy to imagine a situation where a fundamental assumption is made with no-one realising that has occurred. Remember that all indulge in thought experiments some even when they are asleep but their true validity, or otherwise, only becomes apparent when contemplation has ceased and the actual thoughts committed to paper and resulting concrete scrutiny. Supposedly, the essence of a good practical experiment is that it should be readily repeatable. It is relatively easy to see how this could be true, but could equally well be untrue, of any thought experiment. Hence, important results derived via thought experiments should always be 
treated with extreme care. In fact, the above mentioned objection of Bohr to the Einstein, Podolsky, Rosen problem might even seem to indicate he felt this thought experiment had little to do with genuine physical problems. Nevertheless, thought experiments provide an extremely useful tool for practical science and as far as the thought experiment leading to the Einstein, Podolsky, Rosen argument is concerned, it is one which has been viewed and examined now over a large number of years and, seemingly, has always led to a genuine problem in physics.

However, while many scientists might have seen Bohr's rebuttal as signalling the close of the argument, some twenty years later another eminent physicist entered the arena by making a genuine breakthrough in the understanding of the issues posed by the Einstein, Podolsky, Rosen article. This was David Bohm who, in 1951 [4], changed the entire setting of the original challenge to quantum theory in a way which made those issues clearer and easier to understand. In Bohm's thought experiment, although two particles were still involved, instead of considering position and momentum, he simplified matters by considering only one variable of physical interest and that was spin, although some might feel this case more complicated in that it involves spin with its less well-known features rather than the seemingly better understood ideas of position and momentum As with the original Einstein, Podolsky, Rosen thought experiment, the two particles were supposed localised at a distance from each other so that spin measurements are totally separated from one another in both space and time, meaning that one cannot influence the other. However, the two particles are entangled so that once the spin on one is found to be "up", for example, the other one must be "down", and this would be the case for all directions. Quantum mechanics, though, claims this not to be the case; the spin in different directions does not have simultaneous reality. Hence, another thought experiment giving results which raise serious questions about quantum theory. It is not without interest to recall the article by Bohm and Aharonov in 1957 [5]. In this article, after a brief review of the original Einstein, Podolsky, Rosen work, it is shown that it involves a kind of correlation of the properties of distant non-interacting systems, which is quite different from previously known kinds of correlation. They then continue by hypothesising a situation which would still be consistent with all experimental data available at the time but would avoid the paradox. Crucially they then draw attention to an experiment already performed, the results of which may be interpreted as providing the "first clear empirical proof that the aspects of the quantum theory discussed by Einstein, Podolsky and Rosen do represent real properties of matter" [5]. The experiment to which reference was made is that by $\mathrm{Wu}$ and Shaknov [6] and which they noted exhibited the spin correlations of Bohm's version of the Einstein, Podolsky, Rosen paradox. They pointed out that the paper argued against the idea that particles are not entangled or that quantum entanglement of particles might dissipate with distance. This view has not been refuted as yet the entanglement of particles is real and 
doesn't dissipate with distance.

The next big step in the ongoing discussion of the important issues raised by Einstein, Podolsky and Rosen was provided by the theoretical work of J. S. Bell in the 1960's. However, one big impetus for that work was provided by comments attributed to Bohm and Aharonov in their 1957 article [5]. In that paper they claimed that a delayed choice would be necessary if experimenters were to determine whether or not the so-called Einstein-Podolsky-Rosen particles behaved in the way Einstein and his two colleagues found unacceptable; or, in other words, experimenters would have to choose which spin direction to measure only after the said particles were in flight. This, they pointed out, would ensure that neither one particle, nor the experimental apparatus used, could send a signal to the other particle.

It was this requirement which was later brought to the forefront of everyone's attention by J. S. Bell in his ground breaking contributions to the debate. As has been pointed out previously [2], Bell wrote two vitally important papers in this particular area of physics. Somewhat ironically, the first of these papers appeared after the second but both appeared in the mid-1960's, some thirty years after the original Einstein, Podolsky, Rosen article was published. In the first of these [7], entitled "On the problem of hidden variables in quantum theory", he tackled the problem of an error in earlier work by von Neumann. Basically, von Neumann had assumed that the expected value of the sum of a number of observable quantities equalled the sum of the expected values of the separate observable quantities. Bell was concerned by this seemingly reasonable assumption in that he knew it was physically indefensible if the observable quantities are replaced by operators which do not commute. In his eventual article, Bell proved that von Neumann's assumption was not appropriate and so all results deriving from it were, at least, questionable. This, of course, meant that the argument concerning the existence of hidden variables in quantum theory was resurrected. It seems almost natural now to feel that, although Bell already knew of the 1935 Einstein, Podolsky, Rosen article, he should turn his attention more critically to its contents [8]. Bell already knew of the various arguments used against this paper but he-possibly alone-recognised something missed by everyone else-Einstein and his two colleagues were correct or, at the very least, had come up with an important truth. Again as has been pointed out previously [2], this wasn't a claim that quantum mechanics was incomplete but that quantum mechanics and Einstein's insistence on locality and realism were incompatible with one another. Bell's result was expressed in mathematical terms which contained inequalities. He suggested that if these inequalities could be seen to be violated as a result of experiments then that would provide evidence in support of quantum mechanics but, if the inequalities were preserved, then that would provide evidence in favour of the thesis put forward by Einstein and his co-workers. This powerful paper quite naturally provoked several highly distinguished experimental physicists to design experiments to test the inequalities. The results of all the experi- 
ments conducted over a period of many years favoured the validity of quantum theory over Einstein's belief in realism and locality.

Bell had set out to use mathematics to show that Einstein's ideas concerning causality and locality are incompatible with the statistical predictions of quantum mechanics. He also indicated that one of the big problems was created by the locality requirement because that requirement means that the results of measurement on one system may not be affected by operations on another system with which it has interacted in the past. Bell's result showed that either the hidden variable ideas were correct or quantum mechanics was, but not both! Of course, it then followed that if quantum mechanics was correct then non-locality must be an actual feature of the world-even if that be only on a microscopic scale. However, it might be worth noting the actual words Bell used in his introduction to this paper;

"The paradox of Einstein, Podolsky and Rosen was advanced as an argument that quantum mechanics could not be a complete theory but should be supplemented by additional variables. These additional variables were to restore to the theory causalityand locality. In this note that idea will be formulated mathematically and shown to be incompatible with the statistical predictions of quantum mechanics."

Considering some of the introductory comments, it is possibly not without interest to note that Bell refers specifically here to the statistical predictions of quantum mechanics. This very mention of the word "statistical" might to some be an admission of some lack of completeness in the theory under discussion; that is, the incompleteness of quantum mechanics. Also, the stressing of the word "statistical" here leads conveniently to the consideration of another approach to the whole problem of the completeness or not of quantum theory.

\section{Work of Kurt Gödel and Lazar Mayants}

In any discussion of completeness, possibly the first work to spring to mind is that of Kurt Gödel who, in 1931 [9], published results which have, ever since, been regarded as of great importance in both mathematical logic and the philosophy of mathematics. The results are summed up in two incompleteness theorems and are concerned with the limits of provability in formal axiomatic theories. The first theorem proves that, in any consistent formal system within which a certain amount of arithmetic may be performed, there will be statements of the language of that system which may neither be proved nor disproved within the system. The second incompleteness theorem then proceeds to show that such a formal system cannot prove its own consistency. On the face of it, there is nothing here to link directly with the issues raised by Einstein, Podolsky and Rosen but the talk of completeness raises questions which might be in need of consideration. Some have wondered, since physics is so dependent on mathematics, if a lack of completeness in mathematics-as seemingly shown by 
Gödel indicates lack of completeness in physics. As far as quantum theory is concerned, it might be wondered quite reasonably if quantum theory is the system and the manipulations carried out constitute the mathematics, in which case Gödel's theorems might be felt to apply, but this is mere speculation. Nevertheless, it seems this is an approach which might reasonably be born in mind by any investigating the cited work of Einstein, Podolsky and Rosen.

In two books published in the 1980's and 1990's [10] [11], Mayants introduced a novel new way of examining several outstanding issues of the then current physical thinking. It is interesting to note that, in the Foreward to the first of these books, Professor Henry Margenau wrote

"In this book he presents a unique, extremely detailed, and embracive version of a subject that has suffered for a long time from numerous internal imperfections. His approach is new and original, the material covered features not only the foundations of the science of probability but also most of its applications, including statistical and quantum mechanics. The key methodological principle underlying the book is of extraordinary significance and deserves special attention."

Considering the origin, this testimony in itself surely seems to suggest taking the contents very seriously and listening carefully to any conclusions deduced using this "new and original" approach. It is also of interest to note a quote by the author in his Preface from the 1959 Russian edition of Dirac's book, The Principles of Quantum Mechanics. Dirac says

"Our notions about the principles of the physical world are changing in the course of time by consecutive stages. We are now at a certain stage but there is no reason to think that this stage is the last one. We can anticipate further changes in the future, which will perhaps be as drastic as in the past."

With these thoughts of Dirac following from the strong recommendation of Margenau in mind, it seems obvious to continue further to explore and consider the basic ideas of Mayants and how they may be utilised to shed further light on the problems existing in physics after the publication of the Einstein, Podolsky, Rosen article as well as those which have emerged since as a result of perusals of that paper.

The first of Mayants' books listed above lays out his approach in great detail and should really be read from cover to cover to gain a full appreciation of his approach and what he is really attempting to do. However, probably two basic concepts are at the heart of all he does; these are the concepts of concrete and abstract objects. In fact, he takes the first of these as the actual starting point for his entire presentation and it is regarded as a primary concept which may not be reduced to any simpler concepts. He perceives concrete objects in the usual, one might say trivial, sense as being quite simply definite, actually existing objects. It is noted also from the very start that such objects are those which are the subject 
of any experimental study. While the concept of a concrete object is relatively straightforward and agrees with our immediate linguistic interpretation of such a named object, that of an abstract object is less so and, as Mayants himself does in his book, is probably best introduced and understood via examples.

For example, to adapt a situation used by Mayants himself, consider an actual room, which would obviously be a concrete object, and suppose it to contain a number of people, which would again be concrete entities, who differ in their sex and age. Hence, this total number of people may be divided into four groups if by age is meant that an individual is either old or young. Then, to quote Mayants, "a young woman in this room" is simply the image of all the concrete persons belonging to the group of young women and since discussing it may only be achieved by ignoring any differences between the concrete members of this group, it may be termed "an abstract object". Again, the notion of "a man in this group" results from ignoring features, including age, of the concrete members of the group and so must be an abstract object corresponding to the concrete persons. Finally, the idea of "a person in this group" results from discounting all the differences between the concrete persons assembled in the room and is, therefore, an abstract object corresponding to all these persons.

Mayants then continues to discuss several other actual examples to further illustrate his thesis before utilising ideas from set theory to come up with a suitable mathematical definition of this notion of an abstract object. Here though the above example will be deemed sufficient as a good basis for the idea which will be used later. Possibly the crucial point about concrete and abstract objects is that, in any experiment, the scientist involved is a concrete scientist who conducts a concrete experiment on concrete objects with the aid of concrete devices and obtains concrete results.

Having noted this seemingly trivial point about concrete and abstract objects, it may be realised that general laws which hold for each concrete object may be felt of as laws valid for the corresponding abstract object also. This notion lies at the basis of the various scientific theoretical disciplines which, in truth, deal with abstract objects. Of course, the correctness, or otherwise, of any conclusions drawn from developments in any such theory must necessarily be checked by experiments involving the corresponding concrete objects. Implicitly, this possibly obvious fact is taken for granted or, probably in most cases, goes unnoticed and is tacitly ignored. In fact, the necessity for recognising the existence of and difference between concrete and abstract objects is rarely noted at all. This final point follows because, in most cases, confusion of a concrete object with an abstract one rarely causes any confusion or misunderstanding. However, it is Mayants' contention that, in some situations, this confusion is totally inadmissible since it can, and does, lead to paradoxes and erroneous conclusions.

The conclusion to follow from the above is that it must be very clearly understood whether concrete or abstract objects are meant in any situation under investigation. If this is not done, a degree of confusion could easily result. As an 
example, consider (as Mayants did) a very simple question such as "How long is an elephant's trunk?" As it stands this question is indeed totally meaningless. This is due to the fact that "an elephant" is an abstract object which has a trunk since any concrete elephant has a trunk but the trunk of the abstract elephant has no definite length since concrete elephants have trunks of different lengths. The question posed only possesses real meaning when it refers to a specific concrete elephant. As with most of the examples cited by Mayants, this one is very simple but well serves to illustrate this seemingly trivial point concerning concrete and abstract objects and their crucial differences as well as their important links.

Before looking at any paradoxes, after this early stressing of the notions of concrete and abstract objects and their respective properties, Mayants proceeds first to give a detailed discussion of what he terms "probabilistics" or, in other words, he talks about basic ideas in both probability and statistics. In this section, though, he also discusses the Lagrange and Hamilton equations so familiar from classical mechanics. Considering what is to follow in a later section of this article as well as the fact that he has dealt at length with the seemingly trivial point about concrete and abstract objects, it is possibly surprising that he restricts his discussion of these two sets of equations to the case of conservative fields and, therefore, to the situation where a potential energy enters the discussion. However, from the point of view of Mayants' text, this proves a totally acceptable course of action to pursue, although it does quite obviously introduce a restriction on the possible use of at least some of the results which follow. Following on from this section, he proceeds to give a careful outline of the fundamentals of probabilistic physics. This includes a resumé of results in classical statistical mechanics and quantum mechanics. The book concludes with a final section devoted to methodological problems and it is here that he applies his mode of thinking, including the crucial notions of concrete and abstract objects, to some of what are regarded as paradoxes in physics - including the Einstein, Podolsky, Rosen problem which, as has been seen already, should not really be seen as being a paradox. Although this book contains so much more of interest and should really be read in its entirety, the basic notions of concrete and abstract objects, which are at the heart of his discussion of the Einstein, Podolsky, Rosen problem and, indeed, of issues raised by Bell's work, have been highlighted sufficiently clearly for progress to be made in addressing these latter two pieces of work from Mayants' point of view.

Put in a nutshell, Mayants feels there is nothing wrong with the reasoning in the Einstein, Podolsky, Rosen paper but the conclusion seems to contradict conventional quantum mechanics which asserts the pair of particles involved is described by a wave-function which gives only probabilistic predictions and simply cannot yield exact values for either position or momentum. Again, it seems to contradict the quantum mechanical understanding that the position and momentum of a particle may have precise values simultaneously. The important 
word here in Mayants reasoning is "seems". His assertion is that there is simply no contradiction - only a confusion between concrete and abstract particles. As he points out, the Einstein, Podolsky, Rosen thought experiment is, like all experiments, just that - an experiment. As such, it must involve a procedure which is carried out using concrete particles. However, quantum mechanics involved probabilities which, in turn, refer to abstract particles. To Mayants, this alone removes any apparent contradiction between the conclusions of the Einstein, Podolsky, Rosen thought experiment and conventional quantum mechanics. To him it reduces to a case of like not being compared with like. This, it must be admitted, is an argument that may well satisfy some but, equally, will certainly not convince many others. Nevertheless, it is a line of reasoning of which all interested in this ongoing controversy should be aware.

Having "disposed" of the immediate so-called problem posed by the Einstein, Podolsky, Rosen thought experiment, Mayants turned his attention to what he seems to have regarded as the most paradoxical inference it revealed. As far as conventional quantum theory is concerned, the actual state of either particle in the pair depends on what physical quantity relating to its partner has been measured and this is not dependent on the distance separating the two particles. If this is so, it should mean that a particle should realise immediately if a measurement has been made on the second particle, regardless of the distance separating them. The particle should realise the result of such a measurement as soon as it is made. However, there may be no interaction between the particles and it is accepted that no signal may pass between them with a speed in excess of that of light. This has provoked thoughts of "action at a distance". Of course, it must always be wondered precisely what is meant here by the "speed of light" since that speed is well-known not to be a constant but something which depends on the refractive index of the medium through which the light is passing.

Mayants continues by noting that quantum theory has nothing to do with measurement in that it is concerned with abstract systems which, in a sense, do not exist but measurement, being an experimental procedure, may only be made on concrete systems. As for the "action at a distance" puzzle, that is really no puzzle at all. After all, a measurement of any quantity on a concrete particle simply reveals the actual value that quantity has. But, since in the Einstein, Podolsky, Rosen case the sum of the moment a of the two particles is fixed, once that of one particle is measured, that of the other follows immediately from the law of conservation of momentum - the distance between the two particles is simply not a factor in the discussion.

In the final analysis though, the Einstein, Podolsky, Rosen thought experiment is just that and, as such, might not be realisable in practice or might require further technical developments before it can be fully realisable practically. Hence, it is conceivable that, at least at some points in time, no inferences deemed controversial are, in fact, directly verifiable. As a result, other experiments, which are realisable in principle, have been proposed. Some of these are a result of 
Bohm's modification of the Einstein, Podolsky, Rosen situations and/or are related to Bell's work. These have been regarded as versions of the Einstein, Podolsky, Rosen experiment but, in reality, they are not for the following reasons.

Mayants' view is that there are really two types of science: that which requires no probabilistic input and probability related science. In the first of these, any theoretical laws applying to an abstract object are valid for any corresponding concrete object at the same time and so the theory may be verified for of these concrete objects. Classical mechanics provides an excellent example of such a case where the mechanical behaviour of each mechanical system may be predicted with certainty.

On the other hand, theoretical laws applying to abstract objects in a probability related science are of a probabilistic nature and may not be verified on a separate concrete object; their verification relies on performing an appropriate statistical experiment on a large number of concrete objects and collecting statistical data. It is seen that definite values of properties of a particular concrete object may not be predicted in principle in probability related sciences. This is true for any probability related area of science including quantum physics.

Mayants' next points out that the Einstein, Podolsky, Rosen thought experiment involves concrete mechanical systems but the concrete systems considered in most of the experiments conducted to test this thought experiment belong to probability related sciences. In his view, the apparent paradoxes in experiments related to Bell's work are really linked with the incorrect identification as special versions of the original thought experiment to be tested. He asserts that a qualitative understanding of the issues involved may be gained simply by considering the strict distinction between concrete and abstract objects.

The next focus of Mayants' analysis is the situation discussed by Bohm. Although many felt Bohm's suggestion helped clarify the issues involved, Mayants indicates the situation considered by Bohm more complicated than that of Einstein, Podolsky and Rosen. This is because the original proposal dealt with well-known quantities, momentum and position, whereas the Bohm suggestion involved spin with its less well-known features. Again though, Mayants' focus remains with the distinction between concrete and abstract objects. As he stresses, whenever measurement is discussed, it is concrete objects which are involved but only abstract objects are involved whenever quantum mechanical matters are under discussion. Hence, in the case of Bohm's suggested experiment, the spin of one concrete particle along some direction, if the spin of its counterpart along the same direction is known, is quite simply determined by the law of conservation of spin. However, this raises the question of whether, or not, it is possible to verify this directly by experiment. The crucial point here is that both measurements must be made on one and the same pair of concrete particles. In any experimental situation it would be vital to avoid confusing particles belonging to different pairs. This would imply that both measurements would need to be made in one random test or, in other words, practically simul- 
taneously. It might be remembered here that a concrete particle has only one spin component which is related to the axis chosen at the time.

The real experiments performed as a result of Bohm's suggestion and Bell's work yielded results which confirmed the belief that two spatially very remote particles belonging to one pair and whose initial total spin was zero have spins of opposite values, regardless of the direction along which they have been measured. Again the question of how one particle could know the result for the other raised its head. Yet again though the answer lies with the fact that a simple conservation law was involved - the law of spin conservation. Later similar real experiments were performed by Aspect [12] involving polarization of photons instead of spin. These led to similar results but again Mayants points out that, in this case, it is just the law of conservation of polarization which is involved.

By introducing a clear distinction between concrete and abstract objects and by drawing on the validity of the various conservation laws, Mayants appears to have cleared up the problems associated for so many years with the Einstein, Podolsky, Rosen thought experiment. But have they? It is undoubtedly the case that many exist who haven't heard of the work and ideas of Lazar Mayants but it is also undoubtedly true that, to many, the issues raised by the famous Einstein, Podolsky, Rosen paper still exist. It is also surprising to some that the 1998 paper by Santilli [13], which offered a different clarification of the whole saga, remains relatively unknown. However, more of that paper and the following work when more recent experimental work has been discussed.

One final word in relation to the ideas of Mayants seems appropriate as, as will be seen later, it is also highly relevant to the work and ideas of Santilli. In his earlier book [10] Mayants, as has been noted already, quoted a passage from Dirac's book on quantum mechanics. He did, though, use a second quote which is, in fact, the final paragraph of the 1958 English edition of Dirac's monograph, again entitled The Principles of Quantum Mechanics, and it is this which seems apt to repeat at this juncture. Dirac said:

It would seem that we have followed as far as possible the path of logical development of the ideas of quantum mechanics as they are at present understood. The difficulties, being of a profound character, can be removed only by some drastic change in the foundations of the theory, probably a change as drastic as the passage from Bohr's orbit theory to the present quantum mechanics.

This powerful statement from such an eminent theoretical physicist surely deserves careful contemplation.

\section{Modern Experimental Developments}

As will be discussed more later, a conference took place via Zoom in September 2020 at which many issues relating to the problems raised by the Einstein, Podolsky, Rosen article were discussed. All the talks may be heard at either 
http://www.world-lecture-series.org/level-xii-epr-teleconference-2020 or https://www.youtube.com/channel/UCwHACC6p2QTrmdbSIz-mPuw/videos.

One talk was given by Gerald Eigen and it concerned an experiment concerned with measurement of the polarization correlation of the two-photon system produced in proton-electron annihilation. The talk may be heard in its entirety on either of the above sites but should be appearing in print in the conference proceedings before too long. This experiment shows, seemingly quite definitely, that the validity of Bell's inequality is confirmed and, therefore, so is the validity of quantum mechanics. However, as the author himself agrees at the conference, this experiment actually confirms the validity of quantum mechanics for point particles under electromagnetic interactions. This restriction must be noted since it implies that this experiment deals with the verification if the basic axioms of the Copenhagen interpretation of quantum mechanics hold - namely it deals with point-like particles under potential interactions.

Recently, the matter has resurfaced with the announcement of experimental results supporting the Einstein, Podolsky, Rosen assertions at Basel [14]. This has provoked further contemplation of this whole issue of completeness and just what it really means. The Basel team noted that the phenomenon dated back to a thought experiment of 1935 and that it allowed measurement results to be predicted precisely but, of course, as mentioned earlier, it must be remembered always that thought experiments are just that thought experiments and such are very difficult to interpret due to the assumptions made not always being totally clear, possibly not even to the originators themselves. However, again as mentioned earlier, via a thought experiment, Einstein, Podolsky and Rosen showed that precise predictions are possible theoretically in certain circumstances. Briefly, such a notion may be explained as follows: two quantum mechanical degrees of freedom are said to be entangled, or non-separable, if the quantum state of one may not be described independently of the other. Hence, if measurements are performed on both, entanglement means that correlations between the outcomes are an inevitable result. Einstein, Podolsky and Rosen pointed out that, for sufficiently strong correlations, local measurements in one region could apparently change the quantum state in a spatially separated region. This was dubbed by Schrodinger [15] as "steering". Put another way, steering results in measurements on one system being used to predict the results of corresponding measurements on the second system with arbitrary precision in principle. Again, to make the position absolutely clear, it was also the case that the two systems could be separated spatially. The resulting paradox is that an observer may use measurements on the first system to make precise statements about the second system, in fact more precise than an observer who has direct access to that second system but not the first.

The Basel team used lasers to cool atoms to a small fraction of a degree above the absolute zero of temperature. At such low temperatures, the atoms are thought to behave completely according to the rules of quantum mechanics and 
form a Bose-Einstein condensate. In this ultra-cold cloud, the atoms collide with one another constantly, causing their spins to become entangled. The researchers involved then took measurements of the spin in spatially separated regions of the condensate. By using high-resolution imaging, they were able to measure the spin correlations between the separate regions directly and simultaneously localise the atoms in precisely defined positions. Hence, in this experiment, the researchers seem to have succeeded in using measurements in a given region to predict precisely the results for another region.

However, while this work appeared covered openly in the popular press and so became known to a wide variety of people, it is of further interest to note that the authors themselves raised more awareness of other similar experimental work in this field. They noted that, as they put it, complimentary to our work, spatially distributed multipartite entanglement has been observed [16], as has entanglement of spatially separated modes [17].

Hence, it is seen that work is afoot in several establishments which is aimed at investigating the interesting phenomenon of "entanglement". In the past, experiments have concentrated on using light or individual atoms to study the predictions of Einstein, Podolsky and Rosen but the Basel group has successfully used many particle systems consisting of reasonably large numbers of particles to observe the behaviour predicted by Einstein, Podolsky and Rosen in 1935. In fact, as commented on in Science, volume 360, in the three articles quoted here, so-called distributed entanglement was achieved in a very challenging setting involving a large number of cold particles. A quick perusal of the articles shows that, in all three, the entanglement was created initially in an atomic cloud which was allowed to expand. Local measurements in different parts of the cloud which were separated spatially showed that the entanglement survived the expansion.

At the time of writing, it is not known if any serious objections to this work have arisen. If such have emerged, the arguments surrounding the issues raised in the Einstein, Podolsky, Rosen article will, no doubt, rumble on. If none has, or does, emerge then it is conceivable that a new era for physics might be opening up since it is surely the case that applications will follow which we all hope will be of benefit to mankind rather than the opposite. The Basel group itself is seemingly already speculating on possible applications of this purely academic research with which they've been involved. One speculation is that the method they've used might be used for entanglement-enhanced imaging of electromagnetic field distributions and also in quantum information tasks, but that is pure speculation at this stage.

One crucial final point needs to be clarified at this juncture and that is concerned with the apparent divergence of result from these two experiments. However, the two do not, in a very real sense, contradict one another. The Basel experiment deals with what might be termed extended particles while that of Eigen is concerned with point particles that, as such, may only sense potential interactions. Hence, the Basel experiment is not in opposition with this work by 
Eigen since it confirms the need for a completion of quantum mechanics for extended particles

The issues raised in this section have been concerned solely with experimentation linked closely with the problems and questions still surrounding that shortish paper of 1935. There was no mention of any further theoretical thoughts on the subject. However, as was mentioned earlier, it is possibly surprising that little has been heard of the 1998 article by Santilli [13] and it is the lack of knowledge of this work which poses a significant question for the scientific community, although, when you read even just the abstract for that paper, maybe some answers become apparent. With talk of such concepts as nonlinear, nonlocal, non-canonical, axiom-preserving isotopies and spin-isospin symmetry and iso-spaces, some will be put off by the implied effort to understand properly what follows in the body of the paper, while others will dismiss the work out-of-hand because it depends crucially on concepts unfamiliar to them. This may be an improper attitude towards proposed new science but many will have forged impressive curricula vitae based on what they regard as well-established concepts and procedures and will be reluctant to jeopardise their personal positions. However, this attitude is completely understandable and these comments should, in no way, be seen as criticism of anyone or any point of view related to this general matter. It does, though, imply a hugely important question for the scientific community when do we agree to examine with a truly open mind, radical new proposals for help in solving age-old problems? It seems there was no difficulty in examining and accepting a wide range of results from Riemannian geometry, as well as the uncertainties introduced by quantum mechanics, into physics and chemistry some one hundred years ago, so why not afford the same respect to hadronic mechanics or are the fundamental results of quantum mechanics to remain sacrosanct even when they don't answer all the important questions facing the scientific community?

These are vitally important questions in general but are particularly apposite when considering the so-called Einstein, Podolsky, Rosen paradox and work related to it. Basically, the Einstein, Podolsky, Rosen claims that quantum mechanics is an incomplete theory because its description of physical reality does not include all elements of reality, while every element of physical reality should be precisely represented in a complete theory. Santilli's new approach has important consequences as far as this argument is concerned. Traditionally, commuting quantities are believed to be independent but, in the so-called iso-topic completion of quantum mechanics, iso-commuting quantities can be mutually interacting, although it should be understood that such interactions are structurally different from those of action-at-a-distance/potential type. Fundamentally, quantum mechanics may be considered an incomplete theory in that it does not contain the element of reality given by the nonlocal structure of interactions expected from the mutual wave overlapping. Hadronic mechanics overcomes this problem. 
It is important to realise though that, as Santilli himself points out, hadronic mechanics is not intended to represent all elements of reality; it is not meant to be a final theory. Physics is, after all, a discipline which will never admit final theories. Hadronic mechanics simply provides one type of completion of quantum mechanics that of axiom preserving type. It might also be noted at this point that Santilli has also shown via his new mathematics that von Neumann's theorem on hidden variables is quite simply inapplicable under isotopies - note, not violated, but inapplicable! He has also established that the oft-quoted Bell's inequality is not valid universally but holds for the conventional form of quantum mechanics specifically.

\section{Santilli's Hadronic Mechanics}

In the preceding few paragraphs, mention has been made of the work and philosophy of Ruggero Santilli. Some unfamiliar terms have been used, some implications have been made. It is now appropriate to examine in detail both Santilli's work as well as the philosophy behind it and background to it. Hopefully, the scientific community will be in a position to both examine and judge openly and unambiguously.

To fully understand and appreciate Santilli's standpoint on the whole issue of completeness of quantum mechanics, it is probably necessary to go back to his starting point. This was when, as a postgraduate student at the University of Torino, he had the opportunity to study some of the original papers written by Lagrange. He immediately found that Lagrange's original notion for his analytical mechanics required the realisation that not all forces are derivable from a potential. He also studied and accepted the Einstein-Podolsky-Rosen argument due to the inability by quantum mechanics to represent energy-producing processes in physics and chemistry because of their irreversibility over time, while quantum mechanical axioms are reversible over time due to the invariance of the Lie brackets under anti-Hermiticity, $[A, B]=-[A, B]^{\dagger}$. During his Ph. D. studies, Santilli read Bohr's rejection of the Einstein-Podolsky-Rosen argument but could not accept it due to Bohr's silence on a number of elements of reality, already known in 1935, which support a suitable "completion" of quantum mechanics. These included the inability of quantum mechanics to incorporate the external terms added by Lagrange to his celebrated analytic equations to represent non-potential forces which are the origin of irreversible processes. Therefore, Santilli decided to dedicate part of his research life to the verification of the Einstein-Podolsky-Rosen argument,.

Hence, the most popular, favoured form of the famous Lagrange equations of analytical mechanics in use today is not the most general because it ignores all non-potential based forces. Incidentally, the derivations of this popular form of the equations also depend on the mass involved being a constant which must make applications in special relativity problematical. At a slightly later time, Santilli also took the opportunity to study some of the original writings of Ham- 
ilton on his celebrated ideas concerning analytical mechanics. He found that Hamilton had essentially the same view as Lagrange and his original formulations ${ }^{*}$ of his mechanical methods also incorporated terms to allow for non-potential based forces. This realisation of unmentioned problems of generality in conventional mechanics, this to include quantum mechanics led Santilli to propose what was possibly his first important contribution in this field; this was the formulation and proof of the following [17]:-

A macroscopic system with forces that are non-conservative and/or irreversible over time cannot be consistently decomposed into a finite number of elementary particles all with solely conservative forces derivable from a potential and, vice versa, a finite number of elementary particles all in conservative conditions cannot consistently yield, under the correspondence principle or other means, a macroscopic system with non-conservative and/ or irreversible forces.

The importance of this result lies first in the fact that it acknowledges the potential existence and importance of non-conservative forces as well as indicating immediately that quantum mechanics, for example, cannot be deemed a universal theory valid for all possible conditions in nature or, in other words, quantum mechanics cannot be regarded as a totally complete theory. This statement does, of course, raise once again the actual meaning of the term "completeness" as applied in quantum mechanics and it might be felt it implies something even more general and far reaching than that implied in the celebrated Einstein, Podolsky, Rosen paper.

The validity of this theorem has serious implications for physical science. Possibly the most important is related to the fact that twentieth century science is based on Lie algebras with antisymmetric brackets $[A, B]=-[B, A]$ that appear in the time evolution of the physical quantity $Q(r, p)$; that is, in

$$
\mathrm{d} Q / \mathrm{d} t=[Q, H]
$$

where $H$ is the Hamiltonian and the brackets are the usual Poisson brackets. Very early in his studies, Santilli noted that, when the external terms mentioned earlier are included, the correct equation is

$$
\frac{\mathrm{d} Q}{\mathrm{~d} t}=[Q, H]+\frac{\partial Q}{\partial p} F=(Q, H)
$$

where $F$ represents the external terms, those not derivable from a potential and $(Q, H)$ a notation introduced by Santilli as an extension to the usual Poisson brackets. This seemingly small change does, though, create difficulties since this inclusion of the so-called external forces results in a violation of some of the conditions which are usually felt to characterise an algebra, specifically the inclusion of the external terms results in a violation for the new bracket $(Q, H)$ to characterize an algebra as understood in mathematics (due to the violation of the right associative and scalar axioms). 
The philosophy adopted by Santilli for the resolution of the impasse was to characterize the time evolution of irreversible systems via new brackets $(Q, H)$ which:

1) verify all axioms to characterize an algebra as understood in mathematics;

2) the said algebra has to be a covering of Lie algebras as a necessary condition to allow a completion of quantum mechanics in the Einstein-Podolsky-Rosen sense;

3) the said brackets must violate the symmetry of the Lie brackets under anti-Hermiticity

$[A, B]=-[A, B]^{\dagger}$ as a necessary condition to represent irreversible systems.

Following an extensive search in European mathematical libraries, Santilli finally located the definition by the American mathematician A. A. Albert [18] of brackets $(Q, H)$ as being Lie-admissible when the attached totally antisymmetric brackets $\{Q, H\}^{*}=(Q, H)-(H, Q)$ verify all the axioms of Lie algebras; and Jordan-admissible when the totally symmetric brackets $\{Q, H\}^{*}=(Q, H)+(H, Q)$ verify all the axioms of Jordan algebras.

Santilli published his Ph. D. thesis in 1967 in two papers, the first [19] on the algebraic resolution of the indicated impasse with the introduction of the Lieadmissible and Jordan-admissible parametric brackets

$$
(A, B)=a A B-b B A=n(A B-B A)+m(A B+B A),
$$

where $a=n+m$ and $b=-m+n$ are non-null positive scalars. As is well known, from the late 1980s on there was the appearance of a very large number of papers on the simpler $q$-deformations of Lie algebras with brackets $(A, B)=A B-q B A$, none of which quoted their origination by Santilli [19] some twenty years earlier.

In the second paper of his Ph.D. thesis [20] Santilli introduced the parametric Lie-admissible completion of Hamilton's equations

$$
\frac{\mathrm{d} r}{\mathrm{~d} t}=a \frac{\partial H}{\partial p}, \frac{\mathrm{d} p}{\partial t}=-b \frac{\mathrm{d} H}{\partial r}
$$

and their operator counterpart

$$
i \frac{\mathrm{d} Q}{\mathrm{~d} t}=(Q, H)=a Q H-b H Q
$$

which provide an approximate representation of the external terms and related irreversible systems. Rather importantly from an historical as well as scientific viewpoint, Santilli showed in this second paper [20] that the totally symmetric component of the Lie-admissible brackets represents Lagrange's and Hamilton's external terms, thereby realizing Jordan's dream that his algebras would one day see physical application.

Following a decade of teaching positions in the U.S.A., Santilli resumed full time work on Lie-admissible formulations at Harvard University in 1977 and succeeded in achieving the following identical Lie-admissible reformulation of the brackets of the classical time evolution of irreversible systems [21] [22]. 


$$
\frac{\mathrm{d} Q}{\mathrm{~d} t}=[Q, H]+\frac{\partial Q}{\partial p} F=\frac{\partial Q}{\partial r} \frac{\partial H}{\partial p}-\frac{\partial H}{\partial r} S \frac{\partial Q}{\partial p}=(Q, H)
$$

which represent all infinitely possible, non-singular external terms $F$ and related irreversible systems for $S=1-\partial Q / \partial p F / \partial H / \partial p$. In the same monographs [21] [22], Santilli introduced the operator image of the above classical Lie-admissible time evolution in the infinitesimal form

$$
i \frac{\mathrm{d} Q}{\mathrm{~d} t}=(A, H)=Q R H-H S Q=A Q\langle H-H\rangle Q
$$

which represents all infinitely possible non-singular external terms $F$ for $R=1, S$ $=1-F / H$, and related finite Lie-admissible group form

$$
Q(t)=e^{(H R t i)} Q(0) e^{(I i t S H)}=U(t) Q(0) U(t)^{\dagger}
$$

showing the clear non-unitary character of the theory $U U^{\dagger} \neq 1$. In the same works [21] [22] Santilli introduced the name of hadronic mechanics to denote a Lie-admissible completion of quantum mechanics for the representation of irreversible processes, while uniquely and unambiguously admitting quantum mechanics for $R=S=1$ (see the subsequent works [23] [24] [25] recent work [26] and review [27]).

Following the achievement of maturity in the construction [23] [24] and verification [25] of hadronic mechanics, Santilli extended the results to the formulation of hadronic chemistry [28] (see also reviews [29] [30] for the study of the following limitations of quantum chemistry:

1) the lack of an exact representation of molecular data when derived from first principles, with deviations of the theory from experimental data on binding energies of the order of $2 \%$ which is a multiple of the thermal energy released in chemical reactions such as that in the formation of the water molecule;

2) the inability to permit accurate thermochemical calculations in energy producing chemical reactions, such as those for the combustion of fossil fuels, due to the reversible structure of quantum chemistry compared to the irreversibility of the indicated chemical reactions;

3) the evident prediction by the Schrödinger equation of quantum chemistry that the identical electrons of valence bonds should repel each other due to their same charge with a repulsive force not overcome by available quantum models of valence bonds, and consequential absence of a quantitative model of molecular structure compatible with experimental evidence;

4) the incorrect prediction that all molecules are paramagnetic from the lack of an attractive force between valence electron pairs;

5) it should be noted that the irreversible Lie-admissible branch of hadronic mechanics and chemistry stimulated considerable interest for the development of at compatible, irreversible statistical mechanics, evidently as an intermediate step toward the achievement of a direct link between mechanics and thermodynamics. Among numerous contributions are J. Fronteau et al. [31] DunningDavies [32], A. Bhalekar [33], and others. An important collection of historical 
as well as more recent works on irreversible non-potential statistical mechanics has been provided by A. Schober [34].

Recall that both Lie algebras and twentieth century applied mathematics have an associative modular structure in the sense that the action to the right on a Hilbert state $H \psi=E \psi$ is equivalent to the action to the left $\psi H=\psi E, E=\mathrm{E}$, thus admitting one single universal enveloping associative algebra. Lie-admissible mathematics, also called geno-mathematics, was conceived by Santilli [35] [36] [37] for the intent of reducing irreversibility to the following primitive bimodular axioms:

1) A modular action to the right $H>\psi=H S \psi=E \psi$ representing motion forward in time and modular action to the left $\psi<H=\psi R H=y E^{\prime}$, representing motion backward in time, irreversibility being then assured whenever $R \neq S$ (or $E \neq E$ );

2) A forward geno-unit $P=1 / S$ and a backward geno-unit $F=1 / R$ with corresponding forward and backward geno-numbers [38] geno-spaces, geno-geometries and geno-differential calculus [39];

3) Bimodular Lie-admissible lifting of the various branches of Lie's theory, including enveloping associative algebras, Lie algebras and Lie transformation groups [23] [24]. To understand the significance of Santilli's Lie-admissible mathematics in physics, recall that non-unitary time evolutions are known to violate causality when formulated in the conventional Hilbert space over a conventional numeric field. A first significance of the Lie-admissible mathematics is to show that causality is fully verified by Lie-admissible time evolutions because they verify no unitarity conditions provided they are formulated on geno-spaces over geno-fields, called geno-unitarity.

Lie-admissible mathematics is nowadays classified into single-valued, multivalued and hyper-valued [40] [41] [42] in the sense that multiplications and other operations produce one single result, or produce an ordered number of results, or verify Vougiouklis hyperstructural laws, respectively. Single-valued Lieadmissible mathematics is recommended for the representation of simple irreversible processes in physics [25] and chemistry [28]; multi-valued Lie-admissible mathematics is recommended for the representation of complex biological processes [40], such as the representation of the growth in time of seashells [43]; while hyper-valued Lie-admissible mathematics has allowed the initiation of a quantitative representation of living organisms [44] as a collection of an extremely large number of extended constituents in continuous entanglement verifying the Einstein-Podolsky-Rosen argument and, therefore, in continuous communications, resulting in an extremely large number of complex interconnections beyond human comprehension that can be solely representable via hyperstructures.

The above methods have been the basis for Santilli's studies of the Einstein, Podolsky, Rosen argument reviewed later and which have seen him offer possible solutions to some of the questions raised concerning the often unstated re- 
strictions imposed nowadays by widely accepted theories of which quantum mechanics is one. Santilli has often pointed out the many positive contributions quantum mechanics has made to scientific knowledge while, at the same time, being concerned by doubts expressed by leading scientific figures of earlier years. A huge impression was left by Fermi [45] who stated that "there are doubts as to whether the usual concepts of geometry hold for such small regions of space (those of nuclear forces)". This is, by itself, an extremely powerful statement by one of the leading scientific figures of his age but is it well-known, do people pay it due attention? The answer to both those questions is probably "No". Santilli also alludes to a statement included in Blatt and Weisskopf's book Theoretical Nuclear Physics [46] in which they speculate on page 31 on the possibility "that the intrinsic magnetism of a nucleon is different when it is in close proximity to another nucleon". In fact, this statement acted as a major spur to Santilli who claims to have produced a complete theory of total nuclear magnetic moments via his so-called hadronic generalisation of quantum mechanics. Whether or not he has achieved this is for the scientific community as a whole to decide but, until his work is read with open minds and properly digested, no final verdict can be sensibly announced. This indicates, once again, the urgent need for a totally open-minded examination of Santilli's work Another major influence was Dirac's concerns as expressed in an earlier quote when discussing Mayants' views and contributions to the debate. He was further influenced by the views of various philosophers of science, especially Karl Popper, who ended up being a strong supporter of Santilli's proposal to construct a covering, or extension, of conventional quantum mechanics.

All these, and more, acted as stimuli for Santilli. However, when considering any problem, Santilli appears driven by an unshakeable belief in the idea that science, in general, doesn't admit complete and final theories, and could not progress without the introduction of some new mathematics. One immediate example illustrating this is provided by Newtonian mechanics, which had been so successful for so long, finding itself regarded as a special limiting case of relativistic mechanics towards the beginning of the last century. Also, Einstein's general theory of relativity brought to the fore in the world of physics new mathematical methods. This new mathematics involved tensors and was reliant on earlier purely mathematical work by such as Riemann, Ricci and Bianchi. Hence, the huge change in physics at the beginning of the twentieth century was accompanied by new mathematics being introduced and used in physics and a well-established theory clearly being seen to be approximate and not final. Accordingly, Santilli turned his attention to producing new mathematics in order to deal with these new problems. To do this, he turned to the work of Marius Sophus Lie for some of his inspiration. After much intellectual effort, Santilli proposed so-called hadronic mechanics which is basically an image of quantum mechanics formulated via several completely new forms of mathematics, termed by him iso-, geno-, and hyper-mathematics, with so-called isoduals for antimat- 
ter. The corresponding iso-, geno-, and hyper-mechanics are then found to represent single-valued reversible, single-valued irreversible, and multi-valued irreversible systems respectively. Fundamentally, hadronic mechanics preserves all the usual laws and principles of orthodox quantum mechanics but represents what might be termed a completion of that subject, as seemingly required by the well-known argument of Einstein, Podolsky and Rosen [1]. It is strongly suspected by many that Santilli's hadronic mechanics genuinely achieves this objective. In fact, in the introduction to one of his books [47], Popper went so far as to make the following assertion:

"I should like to say that he (Santilli)—one who belongs to a new generation seems to me to move on a different path. Far be it from me to belittle the giants who founded quantum mechanics under the leadership of Planck, Einstein, Bohr, Born, Heisenberg, de Broglie, Schrödinger, and Dirac. Santilli too makes it very clear how greatly he appreciates the work of these men. But in his approach he distinguishes the region of the arena of incontrovertible applicability of quantum mechanics (he calls it atomic mechanics) from nuclear mechanics and hadronics, and his most fascinating arguments in support of the view that quantum mechanics should not, without new tests, be regarded as valid in nuclear and hadronic mechanics, seem to me to augur a return to sanity: to that realism and objectivism for which Einstein stood, and which had been abandoned by those two very great physicists, Heisenberg and Bohr".

However, the whole truth will be known only after the wider scientific community has examined the veritable mountain of material with an open mind. Incidentally, the names for these three new branches of mathematics/mechanics were constructed for the following reasons: firstly the "iso" prefix, being short for isotopic which comes from the Greek and is meant to indicate the property of axiom-preserving for the new theory; secondly, the "geno" prefix comes from genotopic which again follows from its Greek meaning which suggests an axiom-inducing property of that new theory; and finally, the term hyperstructural basically arose from ideas of multivalued functions. Further, iso-mechanics is fundamentally a non-unitary theory but is reversible; geno-mechanics preserves this property of non-unitarity but introduces ideas of irreversibility; hyper-mathematics goes even further and, while preserving non-unitarity and irreversibility, introduces multi-valuedness which increases the number of degrees of freedom open to the investigator and thus permits the study of far more complicated structures than was allowed previously. However, as far as the present discussion is concerned, only Santilli's aforementioned iso-mathematics is strictly relevant. To cover all aspects of even iso-mathematics and the related iso-mechanics in one article would be an impossible task. However, below an attempt will be made to give a flavour of the entire theory with special emphasis on those topics directly relevant to the specific issue under discussion here - the 
Einstein, Podolsky, Rosen proposals from their 1935 article.

\subsection{Santilli's Iso-Mathematics}

From the very beginning Santilli recognised the wonderful success and consistency of traditional quantum mechanics but also noted clear limitations in its range of applicability such as the fact that not all of nature can be reduced to isolated points because of interactions due to wave-overlapping (at mutual distances smaller than the coherent wavelength of each pair) under which quantum mechanics is exact no longer. Therefore, he began a search for generalised methods which gave the traditional theory as a particular case when the mutual distances are such as to render all non-quantum mechanical effects ignorable. The so-called isotopies, which are maps (or liftings) of any given linear, local, unitary structure into the most general possible nonlinear, nonlocal, non-unitary forms but which reduce to the original form in special circumstances, were the result. The isotopies are axiom preserving because of the latter property. The basis for these generalised methods lies in a generalisation of the notion of number and, ultimately, this relies on a generalisation of the whole idea of the unit which, until these suggestions of Santilli, has always been +1 . More generally, everything revolves around the generalisation of the usual basic $n \times n$ dimensional unit given by $I=\operatorname{diag}(1,1,1, \cdots)$ into an $n \times n$ matrix $\hat{I}$ which is well-behaved, non-singular and Hermitian but whose elements have an arbitrary dependence, which is both nonlinear and nonlocal in general, on all required quantities and their derivatives of arbitrary order, such as coordinates $r=(x, y, z, \cdots)$ and wave-functions $\psi(t, r)$ as well as time, local temperature, local density, etc. Hence, the starting point is the mapping

$$
\boldsymbol{I} \rightarrow \hat{\boldsymbol{I}}
$$

which is termed isotopic lifting or lifting for short. More detail for all of what follows may be found in the original papers [48] by Santilli as well as in monographs [23] [24] he wrote subsequently.

In order for his new theory to preserve the basic abstract axioms of conventional methods, Santilli introduced the additional lifting of the conventional associative product of two quantities $A$ and $B$ as follows:

$$
A \times B=A B \rightarrow A \hat{\times} B=A \times \hat{T} \times B
$$

where

$$
\hat{I}=\hat{T}^{-1} \text {. }
$$

Then, it is seen that $\hat{I}$ is the correct right and left unit of the new theory:

$$
\hat{I} \hat{\times} A=\hat{T}^{-1} \times \hat{T} \times A=A=A \hat{\times} \hat{I}=A \times \hat{T} \times \hat{T}^{-1},
$$

where $\hat{I}$ is called the iso-unit and $\hat{T}$ the isotopic element.

In those same original articles cited above, it was also shown that the above two maps lift an associative algebra $\xi$ with the usual unit 1 , elements, $A, B, \ldots$ and a conventional associative product $A \times B$ in an axiom preserving manner 
into a new algebra $\hat{\xi}$ with a new unit $\hat{I}=\hat{T}^{-1}$, and new product $A \hat{\times} B=A \times \hat{T} \times B$. Also, as with the original, the new product is associative:

$$
\xi: A \times(B \times C)=(A \times B) \times C \rightarrow \hat{\xi}: A \hat{\times}(B \hat{\times} C)=(A \hat{\times} B) \hat{\times} C .
$$

It was shown also [21] that the original and new algebras are locally isomorphic under the condition of positive definiteness $\hat{I}>0$, but are anti-automorphic if $\hat{I}<0$. Since the associative law continues to hold at the isotopic level, $\hat{\xi}$ is termed an iso-associative algebra.

When examined in detail, it is seen that, in conventional dynamics, the antisymmetric part $\xi^{-}$attached to the associative algebra $\xi$ with the familiar product $[A, B]=A \times B-B \times A$, which is characteristic of a Lie algebra and results in the time evolution $i \mathrm{~d} A / \mathrm{d} t=[A, H]$, where $H$ is the Hamiltonian, with an exponentiated form $A(t)=\exp (i H t) \times A(0) \times \exp (-i H t)$, constituting a one parameter Lie group.

The isotopies briefly discussed already allow a generalisation of this. The anti-symmetric algebra $\hat{\xi}^{-}$attached to the iso-associative algebra $\hat{\xi}$ leads to $[\hat{A, H}]=A \hat{\times} B-B \hat{\times} A$ which was proved to preserve the Lie axioms at the isotopic level. The fundamental dynamical equations of the isotopic theory are then given by

$$
i \mathrm{~d} A / \mathrm{d} t=[\hat{\hat{y}} H]=A \hat{\times} H-H \hat{\times} A=A \times \hat{T} \times H-H \times \hat{T} \times A
$$

with an exponentiated form

$$
A(t)=\exp (i H \times \hat{T} \times t) \times A(0) \times \exp (-i t \times \hat{T} \times H),
$$

where $\hat{T}$ retains its earlier meaning.

The above constitutes an extremely brief outline of the structural elements of the isotopies of Lie's theory and also serves as a brief introduction to the methods introduced by Santilli in a veritable library of articles and books in an attempt to combat at least some of the inadequacies he observed-as had others before and after him in modern science. The latter equations above are highly nonlinear, nonlocal and non-Hamiltonian but, in isotopic spaces have been shown to retain the conditions of linearity, locality and canonicity. All this is based purely on a generalisation of the basic unit of conventional methods. However, for consistency, it proves necessary for all conventional mathematical methods to be lifted (to use Santilli's own terminology). This would include lifting numbers, fields, angles, differential calculus, trigonometric and hyperbolic functions, special functions and transforms, vector, metric and Hilbert spaces, algebras, geometries, mechanics, etc, This has obviously been an enormous task which has been accomplished by Santilli over a lifetime of endeavour and obviously only a brief resumé can be included here. Nevertheless, an attempt will be made to cover all necessary aspects of this mammoth work by liberal use of references for any interested in the minute detail of required manipulations so that more will be able to follow the reasoning behind his resolution of problems posed by the Einstein, Podolsky, Rosen article. 
The above discussion hopefully gives a brief outline of the sort of terminology and manipulations that are required in the new mathematics underlying Santilli's approach to so many issues facing modern day physics; an approach based totally on the belief that further progress will only be made if new mathematics is introduced. This conviction was based, no doubt, on the way in which people like Newton and Einstein approached the resolution of the theoretical problems of interest to them. While in the above an introduction to the iso-product and to a generalisation of Lie algebra has been discussed, several other definitions and applications are necessary for a truly full understanding of this new mathematical topic of iso-mathematics but, rather than include all the mathematical details here, brief introductions accompanied by original references will be included. This approach is for several reasons, one of which is to not include too much mathematical detail which could easily interrupt the fundamental narrative and secondly too much mathematical detail could detract from the main issue which is the physics involved. The other issues of relevance concerning iso-mathematics are

1) The application to the solution of the Lorentz problem; that is, the invariance of locally varying light speeds $C=c / n$. It must always be remembered that Einstein's assumption was simply that the speed of light in a vacuum is constant, not that the speed of light is constant since, as is well-known, the speed of light does vary in different media. This problem is studied in detail in chapter 8 of [23].

2) As mentioned earlier, it also proves necessary to extend the idea of numbers and, to this end, Santilli introduced the notion of iso-numbers and the details are worked out in chapter 2 of [23].

$3)$ In chapter 3 of [23], the idea of iso-spaces is introduced and discussed in detail while so-called iso-functions are examined in chapter 6 of [39].

4) Iso-differential calculus was first introduced in [39] but it might be helpful to some to consider [49] also.

5) The simple construction of iso-mathematics via non-unitary transforms, together with the important topic of the invariance of isotopic elements and iso-units under iso-unitary transforms are dealt with in detail in [50].

With all this, and more, in place, attention turned to developing what is now referred to as iso-mechanics and, following that the generalisation or, as Santilli terms it, covering of quantum mechanics termed hadronic mechanics. The full details of the construction of this may be found in [23] and [24]. Suffice it to say that hadronic mechanics is based on some fundamental structures:

a) An enveloping algebra $\hat{\xi}$ with generic elements $\hat{A}, \hat{B}, \cdots$ (which are the same polynomials in $r$ and $p$ of the usual quantum algebra only written in iso-space) called the iso-associative envelope and characterised by the iso-associative product:

$$
\hat{A} \hat{\times} \hat{B}=\hat{A} \times \hat{T} \times \hat{B} \text { with isounit } \hat{I}=\hat{T}^{-1} ;
$$

b) The iso-fields $\hat{C}(\hat{c},+, \hat{x})$ of iso-complex numbers $\hat{c}=c \times \hat{I}$, or its iso-real particularisation $\hat{R}(\hat{n},+, \hat{x})$; and 
c) The iso-Hilbert space $\mathfrak{W}$ with isostates $\hat{\psi}, \hat{\phi}, \cdots$ and iso-inner product over $\hat{C}$

$$
\hat{\psi} \uparrow \hat{\phi}=\langle\hat{\psi}|\times \hat{T} \times| \hat{\phi}\rangle=\hat{I} \times \int \mathrm{d} \hat{x}^{3} \hat{\psi}^{\uparrow} \times \hat{T} \times \hat{\phi} \epsilon \hat{C}(\hat{c},+, \hat{x})
$$

The isotopies of both the Schrödinger and Heisenberg representations are then identified. Eventually after some further manipulations it is found that, as a general rule, hadronic mechanics preserves all the axioms of quantum mechanics and actually quantum and hadronic mechanics coincide for all isotopies for which $\hat{I}>0$ at the abstract level. Hence, the axiomatic consistencies of hadronic mechanics are guaranteed and it is evident that hadronic mechanics merely provides a more general nonlinear, nonlocal and non-potential realisation of the same axioms as traditional quantum mechanics. It is, in short, a more general theory of which the traditional one might be viewed as a special case dealing with a restricted number of situations. Hadronic mechanics was conceived and constructed precisely to allow detailed investigations of physical situations which lie outside the scope of traditional quantum mechanics. Having been constructed though, it also allowed further perusal and a deeper understanding of issues raised by the aforementioned article by Einstein, Podolsky and Rosen [1], as well as possible resolutions of other problems which had been outstanding in science for many years. Further, having been deeply interested in environmental issues since his early years in science, it allowed Santilli to put forward other ideas aimed at at least alleviating some of these problems if not actually solving them completely.

\subsection{Resolution of Einstein, Podolsky, Rosen Issues}

The above discussion of the new mathematics devised by Santilli is indeed a very brief introduction as it is meant to be. As stated earlier, anyone truly interested in an in depth examination of these totally new ideas should consult the enormous library of books and articles devoted to it, probably starting with [23] and [24] cited here. It was in a publication of 1998 [13] that Santilli made his first contribution to the Einstein, Podolsky, Rosen debate. In this article, he concerned himself with examining isorepresentations of Lie-Isotopic $S U(2)$ algebra. However, he ended the paper with a discussion of applications of the preceding theory to nuclear physics and, possibly more importantly in the present context, to issues concerning local realism. He proved under the conditions imposed by his earlier mathematics that Bell's inequality and the von Neumann theorem are inapplicable under isotopies and this allowed a limited completion of quantum mechanics along the lines of that envisaged by Einstein, Podolsky and Rosen. It is possibly worth noting at this point that this approach from as long ago as 1998 immediately brings to mind the prediction of Dirac in the final paragraph of his celebrated book on quantum mechanics and quoted earlier when discussing Mayants' approach and ideas. However, for completeness, it is quoted in full here again: 
It would seem that we have followed as far as possible the path of logical development of the ideas of quantum mechanics as they are at present understood. The difficulties, being of a profound character, can be removed only by some drastic change in the foundations of the theory, probably a change as drastic as the passage from Bohr's orbit theory to the present quantum mechanics.

There can be no doubt that Santilli did, and does, provide a truly "drastic change" in the theory due to his new mathematics. Is he correct? Only time will give the real answer to that query but his above mentioned article is only the beginning, not the end, of his involvement with this issue which has plagued theoretical physics for more than eighty years.

One other issue that has always concerned Santilli is that most contemporary theory is, and was, concerned with point particles or, sometimes, particles exhibiting spherical symmetry. He wanted to extend ideas to encompass extended particles and, in an article of 2019 [51], he continued his study of the ideas initiated by Einstein, Podolsky and Rosen according to which quantum mechanics could be (to use their words) "completed" into a much broader theory which recovered classical determinism. What he achieved in this article was to show, by using the previously achieved isotopic lifting of applied mathematics into iso-mathematics and quantum mechanics into the isotopic branch of hadronic mechanics, that extended particles seem to approach classical determinism in the interior of hadrons, nuclei and stars and also appear to recover it in the limiting conditions of gravitational collapse. As in so much of his work, Santilli stresses again in this paper that he is concerned with extended particles immersed in hyperdense media with ensuing linear and non-linear, local and non-local, Hamiltonian and non-Hamiltonian interactions. Yet again it might be noticed the reference to non-Hamiltonian interactions; something missing or, at least, hidden from so many modern discussions of mechanics but which caught his attention as a research student in Italy and has remained with him throughout his professional life. The main result, though, of this article was to show that the standard deviations of coordinates and momenta for particles in hyperdense media are characterised by the isotopic element which, being very small always, $\hat{T} \ll 1$, reduces the uncertainties in a manner inversely proportional to a non-linear increase of the density, pressure, temperature and other characteristics of the medium, while allowing $\hat{T}=0$ under exteme limiting conditions with the resulting recovery of full determinism as predicted by Einstein, Podolsky and Rosen.

As is seen, Santilli appears to have returned to the problems raised by Einstein, Podolsky and Rosen after a lapse of some twenty years and one must wonder if the experimental results published by the Basel group had any effect. Around the time of this 2019 article, Santilli began organising an international conference, to be held in Florida in September 2020, devoted to the issues raised by the Einstein, Podolsky, Rosen article. In the event, the meeting had to take 
place via Zoom and, as was mentioned earlier, for anyone interested in what transpired, all the talks have been recorded and are available from the World Lecture Series website as well as from a YouTube link with the full proceedings are to appear in written form soon.

This conference coincided with the appearance of a further series of three papers published in 2020 and which mark the end of at least this stage of the development of Santilli's ideas on the subject. However, as he himself stresses continually, there can never be a true end to any scientific investigations, only something that seems to be the end at a precise moment in time a possible end dictated by the extent of appropriate knowledge at the time. The three papers in question [52] [53] [54] bear a joint title Studies on A. Einstein, B. Podolsky and $N$. Rosen argument that "quantum mechanics is not a complete theory" but each bearing its own explanatory sub-title. In the first of these [52], subtitled Basic Methods, Santilli begins by restating the fundamental notions put forward in his 1998 [13] paper; that is, he showed that the objections to the Einstein, Podolsky, Rosen position were indeed valid for point-like particles in a vacuum (so-called exterior dynamical systems) but, for extended particles in hyperdense physical media (so-called interior dynamical systems) those same objections are inapplicable note not violated but simply inapplicable since the latter systems seem to admit a classical counterpart when examined within the isotopic branch of hadronic mechanics. Again, as noted earlier, within a more recent article [51], he showed that quantum uncertainties associated with extended particles seem to tend to zero in the interior of hadrons, nuclei and stars and to be identically so in the limit of gravitational collapse. In this first paper of the mentioned trilogy, he went on to review, upgrade and specialise the basic mathematical, physical and chemical methods required in a further detailed analysis of the Einstein, Podolsky, Rosen issue. The details of the work included in this piece are largely mathematical but it is claimed that what was achieved was;

1) A review and upgrade of the so-called Lie-admissible and Lie-isotopic "completions" of twentieth century applied mathematics for the representation of time irreversible and reversible interior systems;

2) A review and upgrade of the "completions" of quantum mechanics and chemistry into the Lie-admissible and Lie-isotopic branches of hadronic mechanics and chemistry;

3) A review and upgrade of the main aspects of the studies to this point; that is, the "completions" of the Newton differential calculus into forms applicable to irreversible and reversible interior systems of extended particles.

Hence, once again, the paper is largely of a mathematical character and it is worth remembering that, in his terminology, "completion" is referring to an extension of existing theory to make the new, more general theory directly applicable to a range of physical topics not covered by presently accepted methods. Also, in this paper he introduces the reader to another class of generalised methods called genotopies, which are a natural generalisation of the isotopies 
when the iso-unit is no longer Hermitian. Again, the mathematical details are included in the text of the article or via copious references therein and it seems appropriate to exclude this mathematical detail here but for the interested reader to follow it up at leisure in detail. The important point in this review is simply to make people fully aware of a mammoth piece of work devoted to extending the scope of theoretical physics and to draw attention to some of the results achieved, specifically, but not exclusively, those connected to issues relating to the Einstein, Podolsky, Rosen paper [1].

In the second paper of this trilogy [53], subtitled Apparent Confirmation of the EPR Argument, he set out to study in detail the iso-symmetries for interior dynamical systems and also confirmed the results of the apparent proof, appearing in his 1998 article [13], that these interior dynamical systems do, in fact, admit classical counterparts. It was also confirmed that the previously published [51] apparent proof that Einstein's determinism is approached progressively for extended particles in the interiors of hadrons, nuclei and stars and is totally verified in the limit of gravitational collapse. All this, as stated here, was simply confirmation of results obtained previously but here looked at afresh and, therefore, worthy of separate consideration since more detail is included to help the reader properly understand and appreciate precisely what has been achieved. However, the paper contained new results as well. For the first time, it is thought, it was shown that the recovering of Einstein's determinism in interior dynamical systems actually implies the apparent removal of quantum mechanical divergencies. This latter point was found due to the rapid convergence of the iso-series of hadronic mechanics, the removal of the singularity in Dirac's delta distribution and other factors which the interested reader can learn about in detail in the original reference. Again, though, it might be noted that everything depends on the validity of Santilli's hadronic mechanics and so, the reference above is to iso-series in hadronic mechanics and not simply series. This seemingly small point is important because it illustrates, yet again, the requirement that allphysical and mathematical quantities involved in all discussions must be those associated with the new language of hadronic mechanics. This may prove an insurmountable obstruction to some but, if the words of Dirac, which appear as the last paragraph of his extremely well-known book on quantum mechanics and which were quoted earlier in full, are to have any true bearing on this vitally important issue for physical science, then getting to grips with this proposed new mathematics is a task large though it may be which should be attempted.

The concluding article of the trilogy [54], subtitled Illustrative Examples and Applications, begins by apparently showing for the first time that the final statement of the Einstein, Podolsky, Rosen article [1] that the wave function of traditional quantum mechanics does not provide a complete description of physical reality, is indeed valid. In point of fact, the claim here is that the study has produced an axiom preserving "completion" of the quantum mechanical wave function due to deep wave-overlapping when represented via iso-mathematics. It 
is shown also that this new approach permits what would otherwise be an impossible representation of the attractive force between identical electron pairs in valence coupling, as well as the representation of the characteristics of various systems both physical and chemical which occur in nature. This "completion" of the usual wave function of quantum mechanics into what might sensibly be termed the hadronic iso-wave function of this new theory also allows the exact representation of all the characteristics of the neutron in its synthesis from the hydrogen atom here a return to the ideas of Rutherford which were deemed incorrect under traditional quantum mechanics. Also, it allows a complete description of the properties of the deuteron again something not achievable with traditional quantum mechanics. A little more will be said about some of these extra achievements in the next section. Although these points may not be felt pertinent as far as a discussion of the Einstein, Podolsky, Rosen issues are concerned, it seems they do fit in in order to give a rounded idea of what is claimed by this totally new approach in theoretical physics and chemistry. Also, all the points to be discussed are, it is felt, of direct relevance to the wider issue of nuclear and particle physics in general.

The above is but a very short introduction to a large body of results produced over a lifetime by someone fascinated by the prospect of extending the range of theoretical physics' theory to cover extended particles but also because of a lack of belief in quantum mechanics as a final theory due to its linearity being at odds with an expectation that the complexity of the Universe cannot always be represented by any linear theory. It is not without interest to note that another scientist who studied non-linear generalisations of quantum mechanics was Heisenberg. In fact, Santilli revealed at the above-mentioned conference that he met with Heisenberg in the early 1970's to enquire about the latter's non-linear theory. The outcome was to discover that, while Santilli was not expecting any non-linear interactions to be derivable from a potential, Heisenberg was seemingly remaining with a Hamiltonian approach dependent on potentials. Nevertheless, the interchange evidently did spur Santilli to proceed to conceive the representation of all non-linear interactions with the isotopic element, in which case the resulting iso-Schrödinger equation does verify the superposition principle and the characterisation of the constituents of a bound state with non-linear internal interactions not derivable from a potential is allowed. Finally, since a quotation from the writings of Dirac has been used more than once already, it is also of interest to note that he, Dirac, was making himself acquainted with some of Santilli's ideas but this possibly interesting and important development was interrupted tragically by Dirac's death.

\section{Some Details of Some Relevant Applications of the Above}

Although not specifically related to matters concerning the Einstein, Podolsky, Rosen issues, it is interesting to note to how many different outstanding problems, including those alluded to above, Santilli has turned his attention with this 
new approach and, seemingly, with so much success. As mentioned earlier, one of his earliest worries concerned the range of applicability of quantum mechanics. Having noted the comments and concerns of some truly notable scientists of the early part of the last century, he devised so-called Hadronic Mechanics and succeeded in explaining a wide variety of otherwise unexplainable phenomena. These are catalogued in detail in his book Foundations of Hadronic Chemistry [28], as well as some being listed here in the abstract, but it is worth noting, and speculating on, some of them here since they, if not all, could well be of interest to anyone concerned with problems in quantum mechanics but, more specifically, in nuclear physics. As noted on page 35 of his book, explaining that the experimental data on the Bose-Einstein correlation in proton anti-proton annihilation at both high and low energy provided experimental verification of hadronic mechanics in particle physics. Such experimental data may be represented by traditional quantum mechanics only after the introduction of arbitrary parameters which seem to have no physical origin. However, hadronic mechanics is easily able to explain things because it proves capable of dealing with the off-diagonal terms appearing in expectation values. This latter property is not allowed in orthodox quantum mechanics because, for a quantity to be observable, its expectation value must be diagonal in form. This, of course, introduces mathematical terms into the discussion which, ideally, should be avoided but, suffice it to say, that the phenomenon may not be explained by orthodox quantum mechanics because it is too restricted as a theory. Another experimental verification, in the sense of the previous example, has been provided by the ability of the new theory to explain data concerning the anomalous behaviour of the mean-life of the kaon with energy. This has been examined successfully over various energy ranges and is important because, as with the example of the Bose-Einstein correlation, it establishes the existence of effects in the interior of kaons which are nonlinear, non-local and, most importantly, non-potential (that is, non-conservative).

As Santilli has stated quite categorically on several occasions but, possibly most clearly at the beginning of section 3 of his article in the Journal of New Energy [55], he has always thought of physical particles as being particles which may be defined rigorously in our spacetime. He points out that hadronic mechanics was conceived and developed in order to identify the constituents of all unstable hadrons with genuine physical particles. Has he succeeded? Time will tell, but the positive evidence is there for all to see and is mounting. As has been seen already, any discussion of this topic inevitably seems to introduce mathematical ideas and notation at some point. Again as stated already, this is unfortunate but doesn't detract from an appreciation of the picture emerging and might serve as a spur for professionals to investigate the detail further in order to reach a truly informed opinion of the work.

From the point of view of physics, it seems that Santilli obtained inspiration from early ideas of Rutherford. It was in 1920 [56] that Rutherford postulated 
the existence of a new particle, which was, in essence a "compressed hydrogen atom"; that is, it was composed of an electron compressed entirely within the proton. This he called a neutron. Presumably Rutherford thought that, when a hydrogen atom is compressed, for example, in the core of a star, the high pressures involved could result in it being reduced in size to that of a proton, with an electrically neutral particle emerging finally. Twelve years later, Chadwick [57] established the existence of the neutron experimentally. However, Rutherford's original conception of this particle was dismissed by many of the founders of quantum mechanics for a variety of seemingly good reasons at the time: the model would require a positive binding energy; both constituents possess spin $1 / 2$ and so, the resulting particle would not be permitted to have spin $1 / 2$ by normal quantum mechanics; orthodox quantum mechanics would also not allow the correct magnetic moment to follow in this model. Hence, the rejection of Rutherford's model of a neutron and this heralded a change in the direction of physics' research. Up to that time, physics had been based on the notion that the constituents of so-called bound states have to be capable of being isolated and identified in laboratories. The rejection of Rutherford's conception appears to have altered this view. This then was the spur for Santilli and, having devised the new mathematics referred to earlier, he first succeeded in producing a consistent model of the meson, $\pi^{0}$, as a bound state of an electron and a positron. This model is not possible in conventional quantum mechanics for a number of reasons, one of which concerns binding energy. Quantum bound states possess negative binding energies and this implies a total mass less than the sum of the constituent masses. For a $\pi^{0}$ meson, this would imply a rest energy appreciably less than its actual rest energy of $135 \mathrm{Mev}$. This problem, as are all others, is resolved by hadronic mechanics or, at least, that is the claim with all the evidence clearly available for examination by those with a mind so to do. The model Santilli proposes does, in fact, explain all the characteristics of the said particle zero spin, electrically neutral, null magnetic moment, a rest energy of $135 \mathrm{Mev}$, a mean-life of approximately $10^{-16} \mathrm{sec}$., a charge radius of about $1 \mathrm{~m}$ (that is, $10^{-15}$ $\mathrm{m})$, decay according to

$$
\pi^{0} \rightarrow e^{+}+e^{-}
$$

and this model of the smallest of hadrons has now been extended successfully to all mesons. Further, although the theory does not view quarks as actual physical particles, but rather as mathematical objects with a composite structure, this new model for hadrons does prove compatible with the current quark theories, always assuming that quarks have a composite structure. For those interested, further details of this model may be found in a variety of publications but especially in volume 4 of the Journal of New Energy [55], as mentioned earlier. In fact this reference is a veritable goldmine of information on this general topic of hadronic mechanics and its consequences both for physics itself and probably for mankind as a whole through its consideration of the possibilities offered by the theory for alternative new clean energies. 
However, what could conceivably turn out to be Santilli's most important achievement was his success in using the new hadronic mechanics to resurrect the Rutherford model for the structure of the neutron successfully. This model recognises a neutron as being composed of a bound state of a proton and an electron at a distance of $1 \mathrm{fm}$; that is, at a distance of $10^{-15} \mathrm{~m}$. As mentioned earlier, such a model is prohibited by conventional quantum mechanics, so, if Santilli's ideas are valid, what are the consequences for physics? The answer is, quite simply, enormous! The abandonment of the original approach to the structure of physical particles would have a profound and far-reaching effect on research in the area of particle physics obviously. However, it is the possible ecological implications which are staggering and of so much direct relevance to absolutely everyone. The orthodox approach has conceivably prevented the study of the neutron as a major source of clean energy and actually seems to have obstructed the study of new forms of clean nuclear energy. These are now being studied via hadronic mechanics, as is the associated problem of the safe disposal of the nuclear waste presently causing so much trouble.

The main characteristics of the neutron, such as its having a rest energy of $939.6 \mathrm{Mev}$, a mean-life of 916 secs., spin $1 / 2$, and a charge radius of $0.8 \times 10^{-13}$ $\mathrm{cm}$., were all explained in a model of the neutron devised by Santilli using hadronic mechanics in 1990 [58]. This was a non-relativistic treatment, but a relativistic treatment soon followed and appeared in 1993 [59]. The crucial point about this is that the model was precisely that proposed by Rutherford so many years earlier. Using hadronic mechanics, Santilli was able to derive all the properties of the neutron when it was viewed as being composed of an electron totally compressed inside a proton. This model, remember, had been abandoned because this structure was inexplicable using orthodox quantum mechanics. However, the fact that the Rutherford model may be explained using this new technique cannot, in itself, be regarded as justification for the new hadronic approach. The real justification is provided by the fact that there appears to be experimental verification of the structure in that experimental verification of the synthesis of neutrons from protons and electrons seems to have been achieved in the 1980's by a group in Brazil under C. Borghi, although the results were published only in 1993 [60]. Although this is exciting, it is by no means conclusive evidence and that is precisely why caution is exercised when reporting and discussing this development. However, the possible ramifications are so important that it is vital for this experiment to be repeated independently several times so that a genuine conclusion may be reached which may be accepted by all in the scientific community.

The ramifications alluded to concern the possibility of utilising these new theoretical ideas to produce new clean energies for mankind. This again is a topic to which Santilli has devoted much time and energy over the years. Basically, many of these new energies are characterised by processes in the interior of hadrons, rather than in nuclei or atoms. It might be noted that energy is re- 
quired if unstable hadrons are to be synthesised from physical particles; in the case of the neutron, $0.80 \mathrm{Mev}$ is required to synthesise it from protons and electrons. However, as Santilli points out [55], "once created, unstable hadrons become a large reservoir of energy, which is released in their decay". Some of these proposed new energies, therefore, are produced by using mechanisms capable of stimulating the decay of unstable hadrons, or by simply using the energy produced in their natural decay. In this article, he goes on to describe the way in which energy could conceivably be produced via stimulated neutron decay. $\mathrm{He}$ also draws attention to the quantity of energy involved, pointing out that the electron emitted in neutron decay would possess energy roughly 100,000 times more than that of electrons hitting a computer screen. Again, it is noted that this mechanism is possible only if the neutron is composed of the physical particles, the proton and the electron. The main ideas behind the proposal are that the neutron does actually decay spontaneously. Also, its mean-life is not fixed but depends on local conditions; for example, if it's a constituent of some unstable nuclei, the mean-life is a few seconds; in a vacuum, it's more of the order of fifteen minutes; in other unstable nuclei, it's even longer; and in natural, light, stable nuclei, it's infinite. However, the neutron itself is naturally unstable and so it is felt it should be possible to stimulate its decay and hence control its mean-life. The actual proposal suggests testing this possibility through the use of photons with the resonating frequency of $1.204 \mathrm{Mev}$, plus the additional threshold energy required to satisfy conservation requirements of

$$
\gamma+n \rightarrow p^{+}+e^{-}+v .
$$

Here the figure of $1.204 \mathrm{Mev}$ for the resonating frequency is another consequence of the hadronic model of the neutron adopted. It has been found, by studying nuclei, that most nuclei do not permit reactions such as that represented by the above equation due to violation of conservation laws. However, some do and it is these which offer the possibility of a new form of usable energy, termed by Santilli hadronic energy. In his book, Santilli chooses, as a representative example, molybdenum $\left({ }_{42} \mathrm{Mo}^{100}\right)$ but also draws attention to the fact that other natural, light elements, such zinc $\left({ }_{30} \mathrm{Zn}^{70}\right)$, possess the required prerequisites. Most of this is still in need of experimental verification. It seems that, if successful, these tests would offer a prize too valuable to be ignored. It is to be hoped, therefore, that the necessary experiments will be performed in the very near future, so that existing doubts may be cleared up, one way or the other, finally.

A further important reason for having the predictions of hadronic mechanics fully and openly tested is provided by the rapid accumulation of highly radioactive nuclear waste around the world. This is proving a major problem for many countries. The U.S.A. has been seen to have a major problem of disposal and also to have an additional problem posed by those opposed to the current method for attempting to achieve that disposal. Britain, on the other hand, while facing problems concerning disposal of its own nuclear waste, faces additional protests from those opposed to its business of helping in the disposal of nuclear 
waste from other countries. In both instances, and in others, people are extremely worried by the perceived threat posed by the actual disposal method as well as that posed by the transportation of that waste across country. All of these worries have been exacerbated by the rapidly growing terrorism threat facing so much of the world. There can be no doubt that a great many people, some with scientific knowledge, some without such knowledge, harbour genuine worries. There can be no doubt also that those worries, and indeed fears, are not unjustified. The above discussion surrounding the composition of the neutron obviously offers the possibility of a resolution of the difficulties and concerns. These essentially reborn ideas concerning the structure of the neutron, if valid, offer the possibility of recycling nuclear waste by way of stimulating its decay in such a way as to reduce the extremely long lifetimes to hours or, at worst, days. It is envisaged that this could be achieved by the use of relatively light equipment and that the nuclear power plants could achieve this within their own boundaries, thus eliminating all transportation of these highly dangerous materials. If the idea works, although jobs in the industry presently formed around the disposal of nuclear waste would vanish, many new jobs in a much safer nuclear waste disposal industry would appear. A new industry might conceivably be expected to grow for the development, production and sale of the new equipment, since it would be a vital requirement for nuclear power plants throughout the world.

The basic idea revolves around the fact that the nuclei concerned are large and naturally unstable. One idea is to expose the highly radioactive nuclear waste to an intense, coherent flow of photons with the required resonating frequency. It is felt that this may be achieved via a synchrotron of about three metres diameter; a size which could be accommodated in nuclear power plants. A typical example is provided by uranium $\left({ }_{92} \mathrm{U}^{238}\right)$ which has a life-time of the order of $10^{9}$ years. A double stimulated transmutation of this element could change it into plutonium $\left({ }_{94} \mathrm{Pu}^{238}\right)$. Again, this is an unstable quantity and has harmful emissions as well, but its life-time is a mere 86 days and it could well be retained under suitable shields for that period of time. It may be superfluous to draw extra attention to this point, but it is worth noting the different life-times involved here 86 days as against $10^{9}$ years! The phenomenal advantage of this stimulated transmutation is immediately evident. Will it work? The theory certainly suggests that it should, but only experimentation will give the actual answer to that question. Possibly the bigger, more relevant, question to ask at this time is whether or not the scientific community and national governments are prepared to finance the experiments necessary to test this thesis?

At this moment in time, it is worth realising that the cost of carrying out the proposed experiments would probably be of the order of a several hundred thousand pounds, maybe even a million pounds. This sounds a lot of money, and indeed it is. However, an experiment to detect neutralinos those particles predicted by theory as candidates for so-called "dark matter" which seems so important to preserve the currently accepted standard model in cosmology has 
been running for many years with little, or no, success so far. Nevertheless, it has been announced that those running this experiment are installing yet another new detector at the cost of one and a half million pounds! It has also been announced that, in America, a new extremely powerful super-computer has been used to create a three-dimensional model of two colliding black holes. Since this is purely a computer experiment, it must be noted from the very outset that any results obtained will be totally dependent on the original input model and information. Both these factors will be completely dependent on present day knowledge and, possibly more importantly, theories. Hence, both will be influenced heavily by "conventional wisdom". Nevertheless, the results from this computer experiment are being heralded as very exciting and it is proposed to use this information to restart another sequence of very expensive experiments to seek evidence of such collisions, including yet another search for gravitational waves. This latter search is again, incidentally, another extremely expensive series of experiments which has continued for a great many years with, as yet, little generally accepted success. This second proposed venture will undoubtedly eat up further millions of pounds of scientific research money. Fundamentally, no-one interested in science should be opposed to either of these two possible areas of research. Both will add, either positively or negatively, to human knowledge and, as such, are important. However, even if successful, neither will produce any immediate major benefit for mankind. If several hundred thousand pounds were to be spent checking out Santilli's theories, the worst that could happen would be negative results; in which case several hundred thousand pounds would have been wasted, but yet again, knowledge would have been gained. Negative knowledge may be, but knowledge nevertheless. If successful though, mankind's energy worries would recede into the background, at least for the immediate future, and nuclear power would become a so much safer option. Also, with the problem of the disposal of nuclear waste dealt with, the genuine worries of so many, when issues surrounding nuclear power are raised, would be assuaged.

However, the scientific establishment tends to regard orthodox quantum mechanics as a sacrosanct part of "conventional wisdom", so it must be thought doubtful that it will sanction work which directly challenges that "foundation stone of modern science". The positions of national governments are far more difficult to assess. They will consult scientific advisers who will be members of the scientific establishment, so the line of their advice is probably predictable. They will be under pressure from a wide variety of areas of "big business" but, no doubt, the most vociferous will be those wreaking profits from the present highly questionable methods of nuclear waste disposal. The individual members of those governments will also, though, be under pressure from members of their electorates. If news of this possibility of there being a truly safe, in-house method of disposing of nuclear waste did become fully public, then it is probably this final factor that would weigh most strongly with national governments 
since, at the end of the day when all the political manœuvering and gesturing has been discarded, it is the thought of votes at the next election which would end up being of paramount importance. Can the possibility of the existence of such a prize really be ignored any longer?

The success in describing the above mentioned model for the neutron using this new hadronic mechanics also opened the way to view afresh models for other systems, in particular the deuteron. Here an unresolved problem had lain around for years; that was the inability of conventional quantum mechanics to explain the value of one for the spin of the deuteron. The deuteron was felt to be composed of two particles, each having spin a half and the basic axioms of quantum mechanics would imply, therefore, a spin value of zero for the ground state of such a system. The new hadronic mechanics clears up this problem also. Following on from the reduction of the neutron to a hadronic bound state of a proton and an electron, the deuteron is viewed as a three-body situation comprising two protons and one electron - or, more accurately in Santilli's language, two iso-protons and one iso-electron. This model is able to represent accurately all the characteristics of the deuteron, including its spin. This success led Santilli to extend the notion to all nuclei. The result was to produce a new hadronic structure model of nuclei in terms of combinations of iso-protons and iso-electrons, which reduces to the usual model involving protons and electrons as a first approximation. This all seems at first sight to be merely another huge amount of almost unintelligible theory which will have little or no effect as far as the ordinary person is concerned. Amazingly, that is not the case. If this theory does turn out to be correct, the implications for society are immense because it could result in a number of new forms of clean energy for mankind's use; forms which are not possible with the old proton - neutron model. It does appear, therefore, that this is an area worthy of further open-minded investigation simply because the possible prize at the end is so attractive and, indeed, necessary considering the massive environmental problems and energy demands facing our world at the moment.

\section{Some Speculative Thoughts}

To begin with one or two quotes from the original paper [1] by Einstein, Podolsky and Rosen which might usefully be brought to the fore:

Firstly, it was stated that

"...every element of the physical reality must have a counterpart in the physical theory."

Secondly, it might be remembered that the authors claimed that

"The elements of the physical reality cannot be determined by a priori philosophical considerations, but must be found by an appeal to results of experiments and measurements"

Which would seem to be an almost self-evident statement if the usual norms 
of scientific practice are to be observed.

They also claimed from their thought experiment that

"by measuring either A or B we are in a position to predict with certainty, and without in any way disturbing the second system, either the value of the quantity $\mathrm{P}$ (that is $p_{k}$ ) or the value of the quantity $Q$ (that is $q_{r}$ )."

Finally, it was stated that

"Previously we proved that either (1) the quantum-mechanical description of reality given by the wave function is not complete or (2) when the operators corresponding to two physical quantities do not commute the two quantities cannot have simultaneous reality. Starting then with the assumption that the wave function does give a complete description of the physical reality, we arrived at the conclusion that two physical quantities, with non-commuting operators, can have simultaneous reality. Thus the negation of (1) leads to the negation of the only other alternative (2). We are thus forced to conclude that the quantum-mechanical description of physical reality given by wave functions is not complete."

Two primary elements of the Einstein, Podolsky, Rosen argument may now be noted separately:

(i) It is possible to define both position and momentum of two previously interacting quantum particles/systems.

(ii) Measurement may not (non-locally) disturb system two if system one is measured, unless a hidden variable not yet defined within the context of wave function is identified.

Point two is clearly implied from the last sentence in the paper:

"We believe, however, that such a theory is possible."

and the aforementioned sentence:

"...every element of the physical reality must have a counterpart in the physical theory."

It is important to note at this juncture, the concerns of Heisenberg regarding such fanciful methods of deduction and exploration as thought experiment and human imagining alone, which appear to closely parallel Einstein's views of the same, as already noted above.

From page 15 of the book on quantum mechanics by Heisenberg [61], concerning the reality of uncertainty, he states that

"In this connection one should particularly remember that the human language permits the construction of sentences which do not involve any consequence and which therefore have no content at all--in spite of the fact that these sentences produce some kind of picture in our imagination; e.g. the statement that besides our world there exists another world with which any connection is impossible in principle, does not lead to any experimental 
consequence, but does produce a kind of picture in the mind. Obviously such a statement can neither be proved nor disproved. One should be especially careful in using the words "reality", "actually", etc., since these words very often lead to statements of the type just mentioned."

This point should be noted as it is of importance for what follows.

If the notion of the Einstein, Podolsky, Rosen argument is sound, it might be expected that the scheme would be used in some sort of demonstrable way. If the idea is good and leads to accurate measurement, some practical usage might have been made of it after all these years. Entangled science aside, is the basic notion in point one above actually demonstrable?

For a moment consider the usual interpretations of the Einstein, Podolsky, Rosen ideas, and imagine two quantum particles which have interacted, and are now moving directly away from each other at a 180 degree relation. This is the interpretation most used, that akin to the thinking of Kumar [62] [63] which defines the Einstein, Podolsky, Rosen idea as "two particles, A and B, [which] interact briefly and then move off in opposite directions."

It might be wondered if this scheme is actually able to measure anything, and is it used? It seems just possible that the answer to this query might be in the affirmative. Positron Emission Tomography scanning (the PET scan) appears to use this idea to measure biological processes and define the locations thereof. A PET scanner is essentially a gamma ray detector. In PET scans, Blood Oxygenation Level-Dependent relations indicative of tissue oxygen metabolism are detected through positron/electron annihilations created by way of an injected radioactive oxygen tracer such as ${ }^{15} \mathrm{O}$, which has a half-life of 123 seconds. As the unstable nucleus of a ${ }^{15} \mathrm{O}$ atom decays having been absorbed by dynamic oxygen using tissues such as neurons, it emits a positron. The positron annihilates when brought in contact with an electron, emitting two (gamma) annihilation photons which travel in exactly opposite directions, a 180 degree relation of two quantum particles moving at a constant mutual speed, allowing accurate measurement of the location of the source interaction in space, and also, inference could easily be drawn from one particle measurement to the values of the other.

It may be concluded that the basic notion is in fact quite functional as a system of measurement when used in a general way. It is clear also that scientific observers could easily infer the position and momentum of one particle from measurement of the other, which travels in an exact mirror opposite direction, both at a known speed.

It does seem, therefore, that the Einstein, Podolsky, Rosen scheme does allow actual measurement, as it should in reality, and is not just a fanciful idea one may draw up to form a picture in one's head, and so, answers in this one aspect at least, both Heisenberg's and Einstein's standards of a workable theory as represented in good science.

Now consider the nonlocal aspects of the Einstein, Podolsky, Rosen theory and assess the outcome of experiments. Local realism insists that measurement 
of one separated system part could not ever superluminally affect the other separated parts of the system (presumably unless some missing, hidden variable is in play). It might be recalled that, in the Copenhagen interpretation of Quantum Mechanics, the wave function is an entirely probabilistic entity! However, it is found that nonlocal measurement effects moving well in excess of light speed are evidenced and those results then repeated in experiments involving entanglement.

In an article by Yin, et al. [64], it may be read:

"In the well-known EPR paper, Einstein et al. called the nonlocal correlation in quantum entanglement as 'spooky action at a distance'. If the spooky action does exist, what is its speed? All previous experiments along this direction have locality and freedom-of-choice loopholes. Here, we strictly closed the loopholes by observing a 12-hour continuous violation of Bell inequality and concluded that the lower bound speed of 'spooky action' was four orders of magnitude of the speed of light if the Earth's speed in any inertial reference frame was less than $10^{-3}$ times that of the speed of light."

How reliable are the results reported in this online article is a question that might come to the minds of many immediately. The answer to that is not known at the present time but, if acceptable, experiments must be repeatable and must be repeated by different personnel. Hence, only time will give the answer to the question. However, in the interim, this all leads to other questions concerning details of the quantum mechanical scenario.

Here, the new theories appear to come good and the possibility arises that the matter may be resolved in favour of a hidden variable: the scalar wave within the aether [63].

\section{Consequences and Further Speculation}

Obviously, if correct, the views of both Mayants and Santilli as laid out above imply serious consequences for physical science as a whole but possibly for traditional quantum mechanics in particular. Mayants lays great stress on the difference between what he terms "concrete" and "abstract" objects, while Santilli points out that traditional quantum mechanics really applies only to point particles and possibly spherically symmetric particles but not to generally extended particles. Using different terminology, both investigators draw attention to the basic limitations imposed in quantum mechanics by the initial assumptions made in constructing the theory. Both in their different ways set out to look beyond those assumptions and see what conclusions might be drawn.

The biggest question raised must surely be concerning the position of the whole idea of uncertainty, especially if Santilli's conclusions are totally valid because they indicate a return to the notion of Einstein determinism or, in other words, indicate a return to the original idea of particles being genuine physical 
objects with genuine physical dimensions and, hence, not hampered by ideas of uncertainty concerning position, momentum, or any other physical property. At this point, the quote from Heisenberg mentioned earlier should be revisited because, in the light of what has just been said, that quote must make anyone wonder if the actual notion of uncertainty is one of those things about which Heisenberg was advising caution. In fact, it appears this well-established property of traditional quantum physics might even be termed a particulate anthropomorphism in that it seems almost as if a human quality, in this case uncertainty, has been assigned to a physical particle. It is perfectly reasonable if such an idea is put forward but it must be asked if it is a human idea or a physical one; it is certainly something which may be pictured but does it really possess physical content?

However, such thoughts immediately raise the question of what could actually be causing the observed measurement results of some well authenticated quantum experiments. If not uncertainty, what is the physical cause of the measurement problem and seeming duality between particle and wave? Of course, if Mayants' reasoning is followed through to its logical conclusion, duality is always the mark of confused thinking as, it might be felt, are most, if not all, paradoxes. Hence, it must be wondered what could be causing the plainly available uncertain effects witnessed in some experiments? Following this line of thought through indicates that there must be some genuine physical reason and it cannot be simply the result of some confused human imagining! This seems to be leading away from direct thoughts about the Einstein, Podolsky, Rosen article but, in truth, is staying very close to it since, if the implications of that article are to have been proved valid, then the consequences are of vital importance to the continued progress of theoretical physical science and, in this, the position of the notion of uncertainty is central. Another consequence must be the existence of so-called hidden variables. Here remember that, in Santilli's approach, he did not claim to disprove any existing results but rather to show that those results were simply not applicable in the extended situations he was considering. Hence, his work has to be considered as an extension to existing theory and not an alternative. One suggestion for coping with this notion of hidden variables in the present context is to resurrect the old idea of an aether. In many ways this notion has not really left science but has often been replaced by the vacuum to which so many different properties have been attributed [65]. Further, more recently, an apparently new solution to the usual Maxwell electromagnetic equations has emerged. An examination of the expressions for the electric and magnetic fields in terms of the usual scalar and vector potentials indicates that a scalar (longitudinal) wave solution exists if the magnetic field is zero but does so whether the electric field is zero or non-zero [66]. These two factors raise the thought that there is a hidden variable which is the aether and longitudinal pressure waves forming up to explain these otherwise puzzling occurrences concerning what appear to be examples of uncertainties. 
If the existence of such an aether is considered for a moment, it might be recalled that boundary layer theory is well established following the initial work by Prandtl [67], the details of which may be found in most books on fluid mechanics such as that by Cole [68]. This theory may then be applied to the aether; that is, the boundary between the aether itself and any body passing through it or over which it passes. Imagine, for a moment, an aetherial boundary layer around a concrete physical particle. It might be argued that the original uncertainty equation describes only effects. The boundary layer as a particle-surrounding scalar wave accounts for the causal mechanism of uncertainty effects, the measurement uncertainty is then caused by an actual wave surrounding the actual particle. In recent times physics has excluded the aether and, hence, the wave around each quantum particle. The uncertainty of the momentum and $x$ component of velocity in the Heisenberg equations themselves might conceivably be caused by this wave obscuring the precise detail of those aspects of the particle. The overall change as diffusion then refers to the heat within the scalar wave and hence its initial (quantum) size, delta in the Heisenberg equations then referring to the amount of change in temperature above absolute zero, in a causal analysis and proper treatment [62]. That wave is the source of "diffusion" effects. Note how, in the paper Entropy in a column of gas under gravity [69], heat first added to the system creates gravitational potential (in part) and not only increase in temperature. That gravitational potential is, by these present theories, the creation of the scalar waves which create a gravitational field.

If this is so, and this, at the present moment, highly speculative theory correct, a violation of measurement "uncertainty" should be observed in experiments if the scalar waves around the particles are deprived of heat. Indeed, this is exactly what is seen in experiments. The back action limit, the quantum limit on measurement precision bounded by uncertainty, is violated, and now, just as might be expected, absolute zero may be approached arbitrarily close to deprive the actual source of the heat needed to create these uncertainty effects. As Clark and colleagues have pointed out recently [70]:

"Here we propose and experimentally demonstrate that squeezed light can be used to cool the motion of a macroscopic mechanical object below the quantum backaction limit. We first cool a microwave cavity optomechanical system using a coherent state of light to within 15 per cent of this limit. We then cool the system to more than two decibels below the quantum backaction limit using a squeezed microwave field generated by a Josephson parametric amplifier."

Uncertainty is experimentally demonstrable as a function of heat instantiated within the boundary scalar wave surrounding the particle. It appears likely that, as heat is further reduced as absolute zero is approached more closely, the cause of quantum uncertainty and fluctuation which is the omnidirectional motion of aether particles within the particle boundary scalar wave is then reduced, perhaps by way of energy reduction of the aether particle itself and/or alignment of 
said omnidirectional particle motions, leading to the absence of any waveforming particulate energy value at absolute zero temperature.

From the point of view considered here it would seem that quantum fluctuation effects and related uncertainty are caused by omnidirectional aether particle motion. Uncertainty itself within quantum particulate measurement dynamics is then actually caused by the boundary wave, surrounding a quantum particle as a function of heat. It might seem also that uncertainty effects emerge as a function of quantum scale, as the aether particle size is more closely approached.

Again, new experiments are seen where, as might be expected, heat is reduced to permit the proliferation of related condensate and Einstein, Podolsky, Rosen effects to emerge. Note, for example the paper by Fadel, et al. [14] referred to in some detail earlier in which it is stated that

"While spin-squeezed and other nonclassical states of atomic ensembles were used to enhance measurement precision in quantum metrology, the notion of entanglement in these systems remained controversial because the correlations between the indistinguishable atoms were witnessed by collective measurements only. Here we use high resolution imaging to directly measure the spin correlations between spatially separated parts of a spin-squeezed Bose-Einstein condensate. We observe entanglement that is strong enough for Einstein-Podolsky-Rosen steering: we can predict measurement outcomes for non-commuting observables in one spatial region based on a corresponding measurement in another region with an inferred uncertainty product below the Heisenberg relation.”

\section{Some Final Thoughts on the Aether}

Before concluding, it might be appropriate to reflect on the demise of the aether theories over the last hundred years and more. In the intervening time, several people have doggedly pursued investigations into theories involving the aether concept, often at personal cost. Among those was Kenneth Thornhill and it might benefit many to read his work which is readily available on the internet [71]. In the cited article, he starts by showing that Planck's energy distribution for a black body radiation field may be derived for a gas-like aether with Maxwellian statistics. The gas consists of an infinite variety of particles whose masses are integral multiples of the mass of the unit particle. Also the frequency of electromagnetic waves correlates with the energy per unit mass of the particles, not with their energy, thus differing from Planck's quantum hypothesis. Identifying the special wave-speed, usually called the speed of light, with the wave-speed in the $2.7 \mathrm{~K}$ background radiation field, leads to a mass of $0.5 \times 10^{-39} \mathrm{~kg}$ for the unit aether particle. Interestingly, in this article he also shows that the speed of light should vary with the square root of the background temperature. It is not without interest to note that this suggestion by Thornhill would obviate any need for introducing theories of inflation to protect the Big Bang notion. More may be found on the whole question of the constancy, or otherwise, of the speed of light 
in the article by Farrell and Dunning-Davies [72].

Also, before ending this section, attention should be drawn to a companion paper by Thornhill [73] in which he discusses in detail the fact that, in a gas-like aether, the duality between the oscillating electric and magnetic fields, which are transverse to the direction of propagation of electromagnetic waves, becomes a triality with the longitudinal oscillations of the motion of the aether if electric field, magnetic field and motion are coexistent and mutually perpendicular. $\mathrm{He}$ points out that it must be shown that, if electromagnetic waves also comprise longitudinal condensational oscillations of a gas-like aether, analogous to sound waves in a material gas, then all three aspects of such waves must propagate together along identical wave fronts. This he shows to be the case. Further he finds that the equations governing the motion and the electric and magnetic field strengths in such an aether, together with their common characteristic hyperconoid, are all invariant under Galilean transformation.

Talk of reintroducing the notion of an aether will be regarded as highly controversial but, if the present indications concerning the validity of the Einstein, Podolsky, Rosen arguments are correct, serious consequences follow for theoretical physical science and, given the discussions relating to physical properties of the vacuum, it seems that thoughts concerning an aether must re-enter the arena of scientific thought.

\section{Conclusions}

For eighty-five years now the arguments of the 1935 article of Einstein, Podolsky and Rosen have continued to cast a cloud over the entire area of quantum mechanics. However, that area of physics has always caused concern in the thoughts and minds of many concerning its real meaning and understanding; after all, accepting the idea of uncertainty as described in quantum mechanics does not come easily to most. Added to this vague doubt probably existing in the minds of many, the Einstein, Podolsky, Rosen article can have done little to dampen these doubts. A further problem with the whole idea must have been provided by the adoption of the so-called Copenhagen interpretation whereby the wavefunction was connected irrevocably with probability because, as soon as probability became a central tenet of quantum mechanics then, at least from a purely linguistic point of view, the theory became incomplete. Linguistically, completeness implies providing a totally exact answer to questions. Hence, in the case of quantum mechanics, that would have meant a theory with the ability to provide exact answers to questions of position, momentum, etc. Once it was clearly acknowledged that this was not the case, the theory became, almost by definition, incomplete. Of course, this argument is based on purely linguistic considerations and it is certainly not inconceivable that another more specific idea of what is meant by "completeness" in a purely physical scientific context is in the minds of some and leads them to claim completeness for the theory.

Here attention has been restricted deliberately to the examinations of the issue 
by Mayants and Santilli, both of which claim the theory incomplete. This has been a deliberate choice since the motivation for writing was provided by the results of the Basel experimental work but this, in turn, led to a perceived need to bring to light theories which, until now, although in the public domain, have been shunned from deep, open-minded assessment by many. The view here is not that these two approaches are necessarily correct but that they are deserving of completely open-minded public examination. Also, as far as the second discussed theory that of Santilli is concerned, further consequences, not strictly within the remit of this general discussion, have been included. These consequences are exemplified by those listed in the abstract, some of which have been examined in more detail in section 6 . This has been to show how the proposed extension to physical science theory, if correct, could have seriously important consequences in a number of areas for each and every one of us.

Further it is suggested here that the notion of "uncertainty" within physical systems is only an anthropomorphic effects descriptor, not a causal description of physics. Apparent fluctuation effects in quantum systems and uncertain measurement effects are in fact caused by a real object and are not the result of probabilistic considerations; it is suggested that one possible explanation could be provided by the existence of an aether and the scalar waves within it. Quantum mechanics as interpreted by the Copenhagen interpretation is, in point of fact, incomplete as stated already. If accepted, this suggestion would imply that the wave function would need to be augmented in its interpretation to include representation of aetherial and scalar wave dynamics. If this were so, it appears that the adjusted theory would conceivably satisfy Einstein's highest standards as a physical theory.

Finally, consider once again the final paragraph of the 1958 English edition of Dirac's well-known monograph, entitled The Principles of Quantum Mechanics:

It would seem that we have followed as far as possible the path of logical development of the ideas of quantum mechanics as they are at present understood. The difficulties, being of a profound character, can be removed only by some drastic change in the foundations of the theory, probably a change as drastic as the passage from Bohr's orbit theory to the present quantum mechanics.

From this, it would seem that Dirac is suggesting, even supporting, the introduction of fundamentally new theory to help in the resolution of difficulties inherent in the subject of quantum mechanics and this in 1958. Possibly the time has come to look afresh at new, possibly revolutionary, ideas and here it would seem appropriate to examine in a totally open-minded fashion the work of such as Mayants and, more importantly, Santilli since it seems distinctly possible that his ideas really do offer a way forward to the above-mentioned suggestion of Dirac. To Dirac it appeared that traditional quantum mechanics had taken theory as far as it could and a radical change to the foundations was necessary if further progress was to be made. It is now time to decide after fair, open- 
minded, public assessment if Santilli's new theories provide at least a first step in achieving that goal.

\section{Conflicts of Interest}

The author declares no conflicts of interest regarding the publication of this paper.

\section{References}

[1] Einstein, A., Podolsky, B. and Rosen, N. (1935) Physical Review, 44, 777. https://doi.org/10.1103/PhysRev.47.777

[2] Aczel, A.D. (2002) Entanglement. John Wiley \& Sons Ltd., Chichester.

[3] Bohr, N. (1948) Dialectica, 2, 312-319. https://doi.org/10.1111/j.1746-8361.1948.tb00703.x

[4] Bohm, D. (1951) Quantum Theory. Prentice Hall, Englewood Cliffs.

[5] Bohm, D. and Aharonov, Y. (1957) Physical Review, 108, 1070. https://doi.org/10.1103/PhysRev.108.1070

[6] Wu, C.S. and Shaknov, I. (1950) Physical Review, 77, 136. https://doi.org/10.1103/PhysRev.77.136

[7] Bell, J.S. (1966) Reviews of Modern Physics, 38, 447. https://doi.org/10.1103/RevModPhys.38.447

[8] Bell, J.S. (1964) Physics, 1, 195. https://doi.org/10.1103/PhysicsPhysiqueFizika.1.195

[9] Gödel, K. (1931) Monatsheft für Mathematik und Physik, 38, 173-198. https://doi.org/10.1007/BF01700692

[10] Mayants, L. (1984) The Enigma of Probability and Physics. D. Reidel Pub. Co., Dordrecht. https://doi.org/10.1007/978-94-009-6294-1

[11] Mayants, L. (1994) Beyond the Quantum Paradox. Taylor \& Francis, London.

[12] Aspect, A. in Lingren, I., Rosen, A. and Svanberg, S. (eds.) (1983) Atomic Physics. Vol. 8, Plenum Press, New York.

[13] Santilli, R.M. (1998) Acta Applicandae Mathematicae, 50, 177-190. https://doi.org/10.1023/A:1005831620903

[14] Fadel, M., et al. (2018) Science, 360, 409-413. https://doi.org/10.1126/science.aao1850

[15] Schrodinger, E. and Born, M. (1935) Proceedings of the Cambridge Philosophical Society, 31, 555-563. https://doi.org/10.1017/S0305004100013554

[16] Kunkel, P., et al. (2018) Science, 360, 413-416. https://doi.org/10.1126/science.aao2254

[17] Lange, K., et al. (2018) Science, 360, 416-418. https://doi.org/10.1126/science.aao2035

[18] Albert, A.A. (1948) Transactions of the American Mathematical Society, 64, 552593. https://doi.org/10.1090/S0002-9947-1948-0027750-7

[19] Santilli, R.M. (1967) Nuovo Cimento, 41, 570-576. https://doi.org/10.1007/BF02902200

[20] Santilli, R.M. (1969) Meccanica, 1, 3-21. https://doi.org/10.1007/BF02129014

[21] Santilli, R.M. (1978) Foundation of Theoretical Mechanics. Vol. I, Springer-Verlag, Heidelberg. 
[22] Santilli, R.M. (1978) Foundation of Theoretical Mechanics. Vol. II, Springer-Verlag, Heidelberg.

[23] Santilli, R.M. (1995) Elements of Hadronic Mechanics. Vol. I, Ukraine Academy of Sciences, Kiev.

[24] Santilli, R.M. (1995) Elements of Hadronic Mechanics. Vol. II, Ukraine Academy of Sciences, Kiev.

[25] Santilli, R.M. (1995) Elements of Hadronic Mechanics. Vol. III, Ukraine Academy of Sciences, Kiev.

[26] Santilli, R.M. (2006) Nuovo Cimento B, 121, 443-486.

[27] Gandzha, I. and Kadisvily, J. (2010) New Sciences for a New Era. Sankata Printing Press, Kathmandu.

[28] Santilli, R.M. (2001) Foundations of Hadronic Chemistry, with Applications to New Clean Energies and Fuels. Kluwer Academic Publishers, Dordrecht. https://doi.org/10.1007/978-1-4020-4004-7 7

[29] Tange, V.M. (2015) Foundation of Chemistry. https://doi.org/10.1007/s10698-015-9218-Z

[30] Trell, E. (2003) International Journal of Hydrogen Energy, 28, 251-253. https://doi.org/10.1016/S0360-3199(02)00031-9

[31] Fronteau, J., Tellez-Arenas, A. and Santilli, R.M. (1979) Hadronic Journal, 3, 130.

[32] Dunning-Davies, J. (2006) Progress in Physics, 4, 24.

[33] Bhalekar, A. (2013) AIP Conference Proceedings, 1558, 702. https://doi.org/10.1063/1.4825588

[34] Schober, A. (1984) Irreversibility and Nonpotentiality in Statistical Mechanics. Hadronic Press, Palm Harbor.

[35] Santilli, R.M. (1979) Hadronic Journal, 3, 440.

[36] Santilli, R.M. (1982) Lie-Admissible Approach to the Hadronic Structure. Vol. I, Hadronic Press, Palm Harbor.

[37] Santilli, R.M. (1982) Lie-Admissible Approach to the Hadronic Structure. Vol. II, Hadronic Press, Palm Harbor.

[38] Santilli, R.M. (1993) Algebras, Groups and Geometries, 10, 273.

[39] Santilli, R.M. (1996) Rendiconti Circolo Matematico Palermo, No. 42, 7-82.

[40] Santilli, R.M. (1997) Isotopic, Genotypic and Hyperstructural Methods in Theoretical Biology. Ukraine Academy of Sciences.

[41] Bhalekar, A.A. and Vougiouklis, T. (2015) AIP Conference Proceedings, 1648, Article ID: 510001. https://doi.org/10.1063/1.4912706

[42] Vougiouklis, T. (2011) Algebras, Groups and Geometries, 28, 83-104.

[43] Illert, C.R. and Santilli, R.M. (1995) Foundations of Theoretical Conchology. Hadronic Press, Palm Harbor.

[44] Santilli, R.M. and Vougiouklis, T. (2020) Algebras, Groups and Geometries, 37. (In Press)

[45] Fermi, E. (1950) Nuclear Physics. University of Chicago Press, Chicago.

[46] Blatt, J.M. and Wiesskopf, V.F. (1963) Theoretical Nuclear Physics. John Wiley, New York.

[47] Popper, K. (1982) Quantum Theory and the Schism in Physics. Hutchinson, London. 
[48] Santilli, R.M. (1978) Hadronic Journal, 1, 223, 574, 1279.

[49] Georgiev, S. (2014) Foundation of the Iso-Differential Calculus. Vols. I to VI, Nova Science Publishers, Inc., New York.

[50] Santilli, R.M. (1997) Foundations of Physics, 27, 1169-1177. https://doi.org/10.1007/BF02551439

[51] Santilli, R.M. (2019) Ratio Mathematica, 37, 5.

[52] Santilli, R.M. (2020) Ratio Mathematica, 38, 5.

[53] Santilli, R.M. (2020) Ratio Mathematica, 37, 71.

[54] Santilli, R.M. (2020) Ratio Mathematica, 37, 139.

[55] Santilli, R.M. (1999) Journal of New Energy, 4, 106.

[56] Rutherford, E. (1920) Proceedings of the Royal Society A, 97, 374. https://doi.org/10.1098/rspa.1920.0040

[57] Chadwick, J. (1932) Proceedings of the Royal Society A, 136, 692. https://doi.org/10.1098/rspa.1932.0112

[58] Santilli, R.M. (1990) Hadronic Journal, 13, 513-515. https://doi.org/10.1002/clc.4960130716

[59] Santilli, R.M. (1993) JJINR Comm., E4-93-352.

[60] Borghi, C., et al. (1993) J. Nucl. Phys. (Russian), 56, 147.

[61] Heisenberg, W. (1930) The Physical Principles of the Quantum Theory. Dover, New York.

[62] Kumar, M. (2011) Quantum: Einstein, Bohr and the Great Debate about the Nature of Reality. W. W. Norton \& Company, New York.

[63] Norman, R. and Dunning-Davies, J. (2018) Hadronic Journal, 41, 1.

[64] Yin, et al. (2013) Bounding the Speed of Spooky Action at a Distance.

[65] Rafelski, J. and Müller, B. (1985) The Structured Vacuum. Verlag Harri Deutsch, Frankfurt.

[66] Freedom of Information Act Electronic Reading Room. https://www.cia.gov/library/readingroom/docs/CIA-RDP96-00792R0005002400016.pdf

[67] Prandtl, L. (1904) Proc. 3rd Internat. Math. Congr.

[68] Cole, G.H.A. (1962) Fluid Dynamics. Methuen, London.

[69] Landsberg, P.T., Dunning-Davies, J. and Pollard, D. (1994) American Journal of Physics, 63, 712. https://doi.org/10.1119/1.17502

[70] Clark, et al. (2017) Nature, 541, 191-195.

https://www.nature.com/articles/nature20604

[71] Thornhill, C.K. (1983) Spec. Sc. Tech., 8, 263.

[72] Farrell, D.J. and Dunning-Davies, J. (2007) New Research on Astrophysics, Neutron Stars and Galaxy Clusters. Nova Science Publishers, New York.

[73] Thornhill, C.K. (1983) Spec. Sc. Tech., 8, 273. 